\title{
COOPERATIVE QUERY REWRITING FOR DECISION MAKING SUPPORT AND RECOMMENDER SYSTEMS
}

\author{
Nader Mirzadeh $\square$ Bell ID, Rotterdam, The Netherlands \\ Francesco Ricci $\square$ Free University of Bozen-Bolzano, Bolzano, Italy
}

This article presents a new technology called interactive query management (IQM), designed for supporting flexible query management in decision support systems and recommender systems. IQM aims at guiding a user to refine a query to a structured repository of items when it fails to return a manageable set of products. Two failure conditions are considered here, when a query returns either too many products or no product at all. In the former case, IQM uses feature selection methods to suggest some features that, if used to further constrain the current query, would greatly reduce the result set size. In the latter case, the culprits of the failure are determined by a relaxation algorithm and explained to the user, enumerating the constraints that, if relaxed, would solve the "no results" problem. As a consequence, the user can understand the causes of the failure and decide what is the best query relaxation. After having presented IQM, we illustrate its empirical evaluation. We have conducted two types of experiments, with real users and offline simulations. Both validation procedures show that IQM can repair a large percentage of user queries and keep alive the human computer interaction until the user information goals are satisfied.

\section{INTRODUCTION}

Business-to-consumer Web sites have proliferated, and nowadays almost every kind of product or service can be bought online. A huge market is available to the end-user through the World Wide Web. Nevertheless, a major problem is generated by this over-abundance of products and services, i.e., how to find the "right" product that satisfies the user needs and wants, which can be like "finding a needle in a haystack."

This problem is only partially addressed by search engines (e.g., Google. com, Yahoo.com or MSN.com, etc.). A search engine can support only the initial stages of the search process, i.e., it can locate Web sites where relevant products are sold. But search engines are keyword-based and are not much useful within a Web site to help the user to identify his preferred

Address correspondence to Francesco Ricci, Piazza Domenicani 3, 39100 Bolzano, Italy. E-mail: fricc@unibz.it 
product in a catalogue. For this specific purpose, recommender systems, a new kind of information search and filtering technology, have been proposed (Resnick et al. 1994; Shardanand and Maes 1995; Resnick and Varian 1997; Schafer et al. 2001; Adomavicius and Tuzhilin 2005; Anand and Mobasher 2005). Recommender systems support personalized search, i.e., they help users to select products when large catalogues are available and the user does not have enough knowledge to select autonomously the best option. For instance, myproductadvisor.com asks a number of questions related to the intended usage of a PC; the user's basic preferences about the PC price, brand, and characteristics; and finally suggests a limited number of options. The well-known book recommender system from Amazon.com suggests to a user books that were bought by users with similar buying behaviors (Linden et al. 2003). In the next two subsections, we shall first briefly discuss recommendation technologies to motivate the introduction of our proposed interactive query management solution, that is discussed in the second subsection.

\section{Recommendation Techniques and Failing Queries}

Recommender systems implicitly assume that a user's needs and constraints can be mapped, by means of appropriate recommendation algorithms, into product selections using "knowledge" managed by the system. Many recommendation techniques have been proposed to automate this mapping (Adomavicius and Tuzhilin 2005; Anand and Mobasher 2005; Burke 2002b) and there are many deployed recommender systems for CD, movies, music, and travel recommendation (MovieLens 2007; Linden et al. 2003; Ricci et al. 2006a). The most popular approach is called collaborative filtering, which recommends products to a user based on his commonalities (e.g., product rates) with other users (Resnick et al. 1994; Shardanand and Maes 1995; Breese et al. 1998; Sarwar et al. 2001). Another commonly used technique is content-based filtering, where the recommendation is based around the analysis of products previously evaluated by a user and the generation of a profile for a user based on the content descriptions of these products. The profile is then used to predict ratings for previously unseen items, and those deemed as being potentially interesting are presented to the user (Billsus and Pazzani 1999; Balabanovic and Shoham 1997; Pazzani 1999).

While, in the former approach (collaborative filtering), a user cannot specify any preferences and is basically pushed by the system with recommendations, in the latter, the user is more in control of the recommendation process since, in addition to the long-term user profile built by analyzing previously evaluated items, many content-based filtering systems allows the user to specify session-specific preferences to further constrain the result set. For instance, in case-based recommender systems, which 
can be considered as a particular type of content-based recommender systems, the user can initiate the recommendation process, providing a set of preferences in the form of a partially defined product description (Burke 2002b; Bridge et al. 2006). In content-based recommenders, the user can actively look for recommendations by querying the underlying catalogue, and the system role is to find products that match the explicit preferences specified by the user and other implicit preferences that may be guessed or derived using the history of previous user-system interactions. Nevertheless, in content-based recommender systems, the search process could often meet two "failure" situations: the system does not have enough information to produce a small set of options, the user request is not satisfiable because some conflicting constraints have been set, or the item repository does not contain exactly the product sought by the user (McSherry 2005).

Among the most interesting approaches proposed to tackle these problems, we mention logical-filtering relaxation, similarity-based filtering, and conversational recommender systems.

Logical filtering imposes the exact match between the query and a set of products. In other words, the query expresses requirements as a set of constraints that must be satisfied. So, there may be situations where no product satisfies the query, and hence an empty result set will be returned. The latter is a major drawback of this technique, and there has been research addressing this problem in the area of cooperative databases (Chu et al. 1996; Gaasterland et al. 1992; Godfrey 1997). Godfrey studied extensively the problem of the empty result set for Boolean queries, i.e., where constraints are on Boolean variables (Godfrey 1997). He has shown that finding, one subquery that returns some results requires a linear time, which is proportional to the number of constraints in the query, but finding all such subqueries make the problem NP hard. Hence, query relaxation in logical filtering can be exploited in real applications only making some simplification of the general problem. For instance, one may search for an approximated optimal solution, where an optimal solution is of relaxing the minimum number of constraints in the lightest way.

Similarity-based retrieval is probably the most frequently used approach, in information retrieval (van Rijsbergen 1979), database (Bruno et al. 2002), and case-based reasoning (Aamodt and Plaza 1994; de Mantaras et al. 2006), to cope with the empty result set problem described. Here a user query partially describes an ideal item/product, and the system retrieves from the catalogue those products that are most similar to the user's ideal product described by the query. The major drawback of this approach is that when no product matches exactly the query, then the ranking induced by the similarity metric "compensates" automatically a match on some attributes with a mismatch on some others. For instance, assume that the user is searching for a hotel with a car-parking facility in the city 
center and at a certain cost. If there is no hotel satisfying all these three constraints, then the similarity retrieval could rank higher those hotels that match the parking and city center requirements, but with a higher cost, and rank lower those with the desired cost and parking, but not located in the city center. But the system could even rank the products in the opposite way. This depends on the relative importance of the features and the particular local similarity measure used for each feature. The user is therefore not helped in understanding what are the available alternatives, in order to take a decision autonomously. In addition, similarity-based retrieval cannot cope with the opposite situation, when a user query matches exactly a large part of the catalogue. The induced similarity-based ranking will rank equally all these exactly matching products.

Conversational recommender systems are characterized by the fact that, when faced with these failure problems, they initiate an interaction with the user in order to refine the current query (Aha and Breslow 1997; Göker and Thomson 2000; Kohlmaier et al. 2001; Gupta et al. 2002; Shimazu 2001). These systems have mostly focused on the large results set problem, and when the user query returns too many results, these systems pose questions to the user to obtain additional preferences to further specify the current query. The questions are determined using feature-selection methods (Doyle and Cunningham 2000; Kohlmaier et al. 2001; Shimazu 2001) that exploit information theory principles (e.g., information gain [Quinlan 1986; Witten and Frank 2000]), to identify the attributes with higher discriminatory power. In other words, they select a feature and generate a corresponding question. When the user replies with a certain value, a new additional constraint of the type "feature-value = user reply" is introduced, and this is supposed to narrow the cardinality of the result set.

Later, we shall discuss some other approaches and the relationships between our methods and those that have already been proposed.

\section{Supporting Recommender Systems with Interactive Query Management}

Considering the benefits and the limitations of both content- and collaborative-based filtering technologies, we have designed a novel hybrid collaborative/content based recommendation methodology, described in Ricci et al. (2003) and Ricci et al. (2006a). In this methodology, a unique human-machine interaction session is modeled as a case in a case-based reasoning framework (Aamodt and Plaza 1994; Bridge et al. 2006). A case collects all the information provided by the user during a recommendation session such as the user's queries to the product catalogues, the selected products, and, in case the user is registered, some stable user-related preferences and demographic data. A recommender system based on this 
methodology models cases and products as two different concepts, and stores them in different repositories (case base and catalogues). This is in contrast with standard case-based reasoning recommender systems that view the catalogue of products as the case base (see Lorenzi and Ricci [2005] and Bridge et al. [2006] for a discussion on that).

All input features provided by the user during an interaction session fall into two (not mutually exclusive) categories: content and collaborative features. Content features are those features that can be constrained in the users' queries, and they are used as descriptors of the products in the catalogues. For instance, in a hotel description the hotel category and the presence of a garage are content features. On the other hand, the nationality of the user or the travel purpose are used to describe the trip, and are not part of any product description found in the catalogue. We have called such features collaborative, since these features are used to measure user similarity within a given session.

When a user searches for a product, she queries the corresponding catalogue in terms of a set of constraints representing the user's needs and preferences. If the query fails to return a manageable set of products, then the user will be assisted by the interactive query manager (IQM) module to refine her query. IQM is a conversational system that manages both the "no result" and "too many results" problems. When finally the user accepts a query, which is suggested by IQM, returning an acceptable set of items, these are ranked using a collaborative-by-content approach (Pazzani 1999), which exploits a double similarity computation (Ricci et al. 2003; Ricci et al. 2006a). The architecture, algorithms, and evaluation of this IQM module is the core contribution of this article, and will be described in the next sections.

In our approach, ranking is based on implicit preferences and is performed after the explicit and attainable preferences are elicited by the user interaction with IQM. Hence, IQM plays a major role in the recommendation process as a conversational and content-based product filtering component.

In this article, we describe the interactive query management component and, in particular, the query relaxation and tightening algorithms. Moving from an initial empirical evaluation (Ricci et al. 2003), which showed the classical entropy-base feature selection technique suggests features not correlated with user interests, we have introduced two new feature selection methods for query tightening. The first assigns to each feature a score proportional to the probability of being used by the user, while the second assigns to a feature a utility value computed by combining the feature information content with the probability of being used by the user. We have therefore compared these two methods with the entropy-based one in two types of simulated user-system interactions. The first one follows a more 
traditional approach that assumes the user will always reply to a posed question about a feature when he has a preference on the values of the feature. Whereas in the second evaluation, we assigned to each feature an acceptance probability that is proportional to the feature popularity measured in real user interactions with the system.

While in the first evaluation, the entropy-based approach outperforms the new methods in term of result-size reduction, in the second evaluation, the situation is reversed and the entropy-based method is outperformed by both the popularity and utility methods. The rationale of this result is that these two new methods select questions/features that will more likely be used to further constrain the result set, and therefore they keep the interaction alive.

Query relaxation is implemented as an approximate linear time algorithm that, if it can find a successful relaxation, the query would be close to a maximal one with respect to the number of constraints kept in the relaxedquery. Thus, since a query represents a user's preferences, the algorithm relaxes as few as possible of the user's preferences. We discuss the properties of the proposed relaxation algorithm, and we show that it is powerful enough to recover from real failure situations most of the time $(86 \%$ of the input failing queries).

At the end of this article, we briefly illustrate two recommender systems where we have applied interactive query management, we discuss some related work, and we summarize our research contributions.

\section{INTERACTIVE QUERY MANAGEMENT}

All major eCommerce Web sites allow users to search in their product catalogues. These Web sites ask the user constraints or preferences related to the products, and retrieve those items that match, exactly or only to certain extent, the user requirements. As we mentioned in the introduction, two basic failure situations may take place in replying to a user query: either too many or no items/products are found. These situations occur quite often, even if sometimes the user is not aware of that because automatic filtering or implicit relaxation approaches, such as those described earlier, are applied. For instance, when too many results match the user's preferences/ constraints, a common "trick" is to present only a few of them, by automatically filtering out some of the results. This filtering technique is typically not personalized, and this will tamper the goodness of the recommendation.

In our view, the available solutions to these problems are not completely satisfactory. The underlying tradeoff between keeping the user interface simple and, at the same time, catching all the user needs, is typically resolved in real applications in favor of the simplicity, i.e., automatically 
solving the problem without involving the user in a dialogue. This is correct but it leaves the space open to more sophisticated solutions that can better balance simplicity with added functionality.

In fact, solutions to these problems can benefit by observing how these interaction problems are managed in real shops via human (customer)-tohuman (clerk) interactions. In this situation, we typically observe long interactions, where questions arise from both the customer and the clerk to make explicit both the search conditions and the offers. In fact, the search constraints are never clear at the beginning, and the initially formulated customer preferences and requirements are iteratively refined, with the help of the clerk. For instance, in a travel agency, a hotel search may proceed as follows.

- Customer: I'd like to find a 3- or 4-stars hotel with the price up to 100 Euros.

- Clerk: There are too many hotels with these characteristics. Would you like to have facilities like car parking or air conditioning?

- Customer: Yes, I need car parking and a restaurant.

- Clerk: I'm sorry but we do not have any hotels with all those characteristics. There are 40 hotels in the desired category with parking and a restaurant, but you have to consider a price up to 110 Euros. There is also one hotel with your desired category and price that has a restaurant, but it has no parking.

- Customer: Let me see this cheap option [she sees it].

- Customer: Let me now see the expensive ones.

- Clerk: Would you like to have an Internet connection in your room?

- Customer: Yes [and she gets 10 suggestions].

What emerges from this dialogue is that the clerk not only replies to the customer (decision maker), but also suggests modifications to the user's requirements (query) to keep the conversation alive and to better converge to a positive outcome, i.e., a user selection. In order to support such types of dialogues, we have developed a technology called interactive query management (IQM) that cooperatively and interactively helps the user to autonomously rewrite a query issued in the context of a decision-making problem supported by a recommender system (Ricci et al. 2006a). The next section describes the logical architecture of IQM.

\section{Architecture of IQM}

Figure 1 shows the logical architecture of IQM. In IQM the user's information needs are encoded as a query. The client application, for instance, a graphical user interface or a recommender component that ranks products, interacts with IQM providing the input query. IQM then displays items that satisfy the query constraints, if any, or otherwise shows 


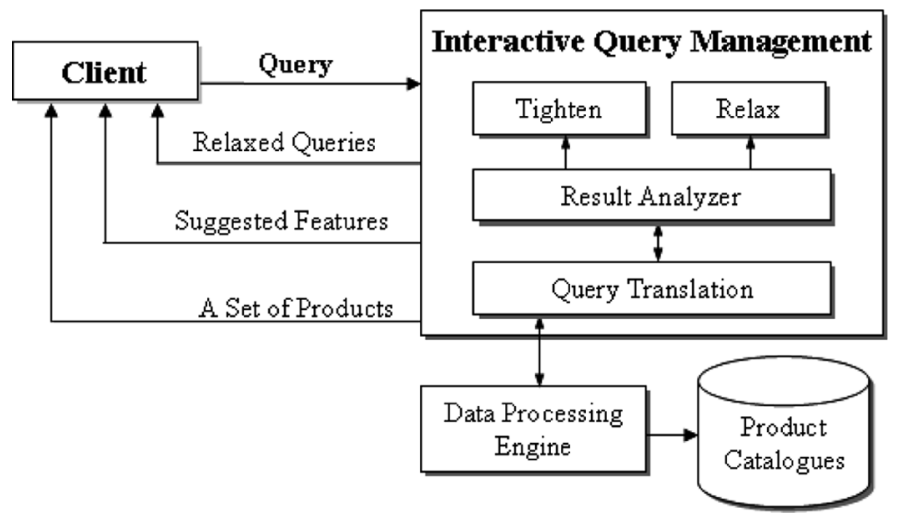

FIGURE 1 Interactive query management architecture.

suggestions to refine the query and collects further input from the user. The data processing engine provides physical data storage, query processing, and some utility functions needed by IQM. When a query is sent to IQM, it is translated (according to some mapping rules) into a format that is processable by the external data processing engine. This engine processes the rewritten query, and sends the required data (e.g., how many items satisfy the query) to IQM to analyze it. At this point, the query result set may contain either: i) no item, ii) a reasonable number of items, or iii) too many items. In the second case, the results are sent to the client. In the latter case, IQM calls the tighten module to suggest a set of candidate features that should be constrained, in addition to those already included in the query. In the former case (i.e., the query has returned an empty result set), IQM invokes the relax module to find all relaxed alternative queries that changing or removing a small set of constraints in the failing query make the query satisfiable, and reports these alternative options.

In the next section, we shall first describe the query language, then the feature selection methods exploited in the tighten component, and then the relaxation algorithm that computes the alternative relaxation suggestions.

\section{Query Language}

The query language should be simple, to be used by a generic user, but powerful enough to let her express the most important information goals. To support dialogues as that shown previously, the customer should be able to express her preferences as a conjunction of constraints (e.g., constraints on price, category, parking, etc.), which the ideal product should satisfy. 
IQM supports a vector-based representation of products, and queries are defined as conjunctions of simple constraints on a single feature. More formally, let $X=\prod_{i=1}^{n} X_{i}$ be a collection type. We shall refer to a feature of $X$ as $x_{i}$, and $X_{i}$ will be called the domain of $x_{i}$, for all $i=1, \ldots, n$. A collection or catalogue $C_{X} \subseteq X$ is a finite set of objects of type $X$. A query $q$ (over the collection $C_{X}$ ) is obtained by the conjunction of atomic constraints; $q=c_{1} \wedge \cdots \wedge c_{m}$ (or simply $q=\left\{c_{1}, \ldots, c_{m}\right\}$ ), where $m \leq n$ and each $c_{j}$ constrains a feature, say $x_{i j}$. Moreover, every $c$ in $q$ has one of the following forms, assuming that $c$ constrains feature $x_{i}$ :

$$
c \equiv \begin{cases}x_{i}=\text { true } & \text { if } x_{i} \text { is Boolean } \\ x_{i}=v & \text { if } x_{i} \text { is nominal } \\ x_{i} \in\left[l_{i}, u_{i}\right] & \text { if } x_{i} \text { is numeric } \\ x_{i}=* & \text { for all feature types }\end{cases}
$$

where " $*$ " is a wild-card symbol and the constraint " $x_{\mathrm{i}}=*$ " is always evaluated as true.

Note that even if the domain of a feature may be infinite such as the real numbers, the feature values occurring in a catalogue are always finite. Hence, there are always the minimum and maximum values for a numeric feature in a catalogue. The range of a numerical feature is defined as $\operatorname{range}\left(x_{i}\right)=U_{i}-L_{i}$, where $L_{i}$ and $U_{i}$ are the minimum and maximum values of $x_{i}$ in the catalogue. In a range constraint, as $c={ }^{l} x_{i} \in\left[l_{i}, u_{i}\right]^{\prime}$, the range of the constraint, range $(c)$, is $u_{i}-l_{i}$, and we have $\left[l_{i}, u_{i}\right] \subseteq\left[L_{i}, U_{i}\right]$.

A constraint is called Boolean, numerical, or nominal if it constrains a Boolean, numeric, or nominal feature, respectively. Let count be the classical function that returns the number of items that satisfy a query $q$ in the catalogue $C_{X}$, and let $\alpha>0$ be an integer. As we mentioned earlier, we shall identify methods to cope with situations when a query fails, i.e., the result set is empty $(\operatorname{count}(q)=0)$ or when the results set is too large, i.e., $\operatorname{count}(q)>\alpha$.

We note that the definition of a constraint on a Boolean feature may seem redundant since a Boolean feature is a nominal one as well, but in such a way we make explicit the fact that Boolean features are constrained only with true values. Let us now consider an example query.

Example 1. The query $q=\{(3 \leq$ category $\leq 4),($ parking $=$ true $)$, $($ price $\leq 100),($ restaurant $=$ true $)\}$ models the query given by the customer at the second interaction step of the example dialogue presented in the previous section.

\section{Query Tightening}

When a query returns many items, the user will either browse the full list or will consider only a subset of items, typically the top ones. A more 
convenient approach would be to help the user to express additional preference(s) she may have and therefore to focus her attention on a smaller and more manageable set of options. Hence, in IQM when a query returns a large result set, i.e., above an application dependent threshold, the system tries to assist the user, to refine the query and build a more specific one. In such situation, IQM suggests some features, three in our recommender systems based on IQM, and waits for the user's action. The user can either select/use some of the proposed features to further specify the query, or ignore the suggestions and browse the full list. In the following sections, we present some feature selection techniques that have been integrated in IQM.

\section{Feature Entropy}

The entropy (or information value) of a feature is a measure of the amount of information provided by the feature for identifying a product (Witten and Frank 2000). Hence, for instance, if we want to find a hotel that has "TV" and "Parking" (Boolean features), then we may want to know if the first or the second feature is more useful in such an identification task. Imagine that all the hotels have a TV but only half of them have a parking facility; it is clear that the "TV" feature is of no use, its entropy is 0 , whereas the second provides one bit of information, separating the set of hotels into two equally populated groups. Feature entropy and a derived measure called information gain have been largely used in building decision trees, as in the ID3 algorithm, to select the best feature to further branch a node in the expansion of a decision tree (Quinlan 1986).

Hence, similarly to its usage in the node selection problem in the induction of decision trees, the entropy of a feature can provide a score useful to rank the features not already constrained by the user. Features with higher scores should be asked to the user because they are the most useful to identify the required product. In fact, many conversational systems (Kohlmaier et al. 2001; Shimazu 2001) have applied this idea. But, in order to apply techniques similar to those used in the induction of decision trees, these systems identify a particular feature as the "class" of the product or consider each product as belonging to a different class. In these systems, when a query has a large result size, the feature having maximal information gain, i.e., the feature that maximally reduces the entropy of the product set, is selected and the user is asked to provide a preferred value for that feature.

The entropy of a feature, say $x_{i}$, on a given set of items, for instance, the result set of a query, is given by the following formula

$$
H_{R_{q}}^{i}=-\sum_{v \in X_{i}} p_{v}^{i} \log \left(p_{v}^{i}\right)
$$


where $R_{q}$ is the result set of $q, X_{i}$ is the domain of $x_{i}$, and $p_{v}^{i}$ is the estimated probability of observing the value $v \in X_{i}$ in $R_{q}$. Here we estimate $p_{v}^{i}$ as

$$
p_{v}^{i}=\frac{\# \text { of items in } R_{q} \text { with } x_{i}=v}{\left|R_{q}\right|}
$$

Filtering a result set by further constraining a feature with high entropy is likely to reduce the cardinality of the result set. The major problem of this approach is that it does not take into account the decision maker interests or preferences over different features. In other words, a feature may have a large entropy in the result set (for instance, the "parking" in the example given previously), but could be of minor interest for the user (he is traveling by train). A better solution would be to integrate the usefulness of a feature with the likelihood that it will be interesting for the user. This observation is at the base of the feature selection methods described in the next sections.

\section{Feature Popularity}

While the feature entropy, presented in the previous section, scores each feature according to a measure of the distribution of values in the repository of items, the method discussed in this section is based on a statistical analysis of the input queries collected by the system. The user queries represent the users' preferences and needs. If a feature has been often used, i.e., it is "popular," then this feature is more likely to fall into the interests of a generic user of the system. More precisely, the popularity score of a feature $x_{i}$ is defined as

$$
\hat{p}_{i}=\frac{\# \text { of queries constraining } x_{i}}{\text { total number of queries }}
$$

The computation of popularity is trivially simple and does not require an online computational effort (at query time), as the entropy does. Its major drawback, when used as a scoring mechanism for feature selection, is that it may suggest features that are not very useful in reducing the result size of the current query, since its suggestion is solely based on users' past queries and does not take into account the feature values distribution. In this example, the popularity method may score high the "TV" feature even if, knowing that the user is likely to be interested in TV, it is not useful in finding a good hotel for him, since all the currently selected hotels do have TV.

We argue that an effective feature selection method must balance "popularity" and entropy, suggesting features that would probably be used by the decision maker, hence keeping the conversation active, and those 
that would likely produce a good reduction of the result size. An approach to combine these two goals is provided in the next section.

\section{Feature Utility}

We present here a third feature selection method that combines the information value, entropy, of a feature with an estimation of the probability of its usage by the decision maker, that is provided by feature popularity. We call this new method utility-based.

Utility theory is a tool for modeling rational decision making (Russell and Norvig 2003). In this setting, the utility function maps world states $S_{1}, \ldots, S_{M}$ to real numbers, $U\left(S_{j}\right)$, expressing the utilities (preferences) of the states. A state is the outcome of an action available to a rational agent $A_{1}, \ldots, A_{N}$. The expected utility of action $A_{i}$ given the evidence $E$ is

$$
E U\left(A_{i} \mid E\right)=\sum_{j=1}^{M} U\left(S_{j}\right) p\left(S_{j} \mid A_{i}, E\right)
$$

where $p\left(S_{j} \mid A_{i}, E\right)$ is the probability of reaching state $S_{j}$ after action $A_{i}$ with the background evidence $E$.

In our model, action $A_{i}$ is performed by the system and models the suggestion of feature $x_{i}$ to the user to further constrain her current query. If an item space has $n$ features, then we have $S_{1}, \ldots, S_{2 n}$ outcome states $(i=1, \ldots, n)$

$$
\begin{aligned}
& S_{2 i-1}=\text { user accepts } A_{i} \\
& S_{2 i}=\text { user rejects } A_{i}
\end{aligned}
$$

When a system suggestion is accepted, the user specifies a preferred value for that feature, and the current query is tightened. In this context, given action $A_{i}$ only states $S_{2 i-1}$ and $S_{2 i}$ can be reached, hence $p\left(S_{j} \mid A_{i}, E\right)=0$ if $j \neq 2 i-1$ or $j \neq 2 i$, and $p\left(S_{2 i-1} \mid A_{i}, E\right)=1-p\left(S_{2 i} \mid A_{i}, E\right)$. Moreover, in this case, $E$ is the evidence about the features already constrained at a given interaction stage.

We define the utility of state $S_{2 i-1}$ as the entropy of feature $x_{i}$ in the current result set. The rationale is that a feature with larger entropy is more likely to reduce the result set if it is constrained. Whereas the utility of state $S_{2 i}$ is 0 , since the system suggestion is discarded by the user. Thus,

$$
\begin{aligned}
& U\left(S_{2 i-1}\right)=H_{R_{q}}^{i} \\
& U\left(S_{2 i}\right)=0,
\end{aligned}
$$

where $H_{R_{q}}^{i}$ is the entropy of feature $x_{i}$, as defined in Eq. (2) 
In order to compute the expected utility of action $A_{i}$, we must compute the probability $p\left(S_{2 i-1} \mid A_{i}, E\right)$. This could be estimated by observing real interactions and measuring how many times the user accepted a system suggestion. A simpler estimate consists in using the popularity of feature $x_{i}$. Hence, we make the simplifying assumption that $p\left(S_{2 i-1} \mid A_{i}, E\right)$ is proportional to the a-priori probability that $x_{i}$ is used in a query, i.e., is proportional to the popularity of the feature $x_{i}$, defined in Eq. (3)

$$
p\left(S_{2 i-1} \mid A_{i}, E\right)=\beta \hat{p}_{i}
$$

where $\beta$ is a positive real constant. The rationale of this estimate is that, if a feature is frequently used/constrained in the users' queries, then it is more likely to be accepted by the user when it is suggested by the system.

It follows from this discussion and Equations (4)-(6) that the expected utility of suggesting feature $x_{i}$ becomes

$$
E U\left(A_{i} \mid E\right)=H_{R_{q}}^{i} \beta \hat{p}_{i}
$$

We finally observe that when sorting a set of features, according to their expected utilities, the $\beta$ constant does not have any influence since $E U\left(A_{i} \mid E\right) \geq E U\left(A_{j} \mid E\right)$ iff $H_{R_{q}}^{i} \hat{p}_{i} \geq H_{R_{q}}^{j} \hat{p}_{j}$

\section{Query Relaxation}

We have already underlined the fact that a query expresses the user (customer) preferences about her ideal product. When a query fails, that is, the result set is empty, then IQM calls the relax module to find a set of relaxed subqueries of the original failing query that returns some results. These subqueries are obtained by relaxing the minimum number of constraints, or in other words, keeping the number of user preferences as large as possible.

The function $\operatorname{relax}(c, \gamma, \delta)$, shown in Figure 2, defines the relaxation operator on a single constraint $c$. This function takes as input a constraint $c$, and two constants $\gamma$ and $\delta$, which are used only when the constraint is numeric. When the constraint is not numeric, the relaxation is trivial; it simply discards the constraint. When the constraint is on a numeric feature, then this is a range constraint $c=(x \in[l, u])$, and $\gamma$ specifies the factor used to decrease the upper bound, and increase the lower bound of the range $[l, u]$ (line 7 and 8 in $\operatorname{relax}(c, \gamma, \delta)$ ). If the resulting change in the constraint range is too small, for instance, when the user has set a very small range in the query, then we use the $\delta$ parameter to force a range expansion, on both sides, at least of size $\delta$. Note that $\gamma$ could be $\infty$, in this case, after the relaxation, the range constraint become $x=[L, U]$, or equivalently $x=*$. 


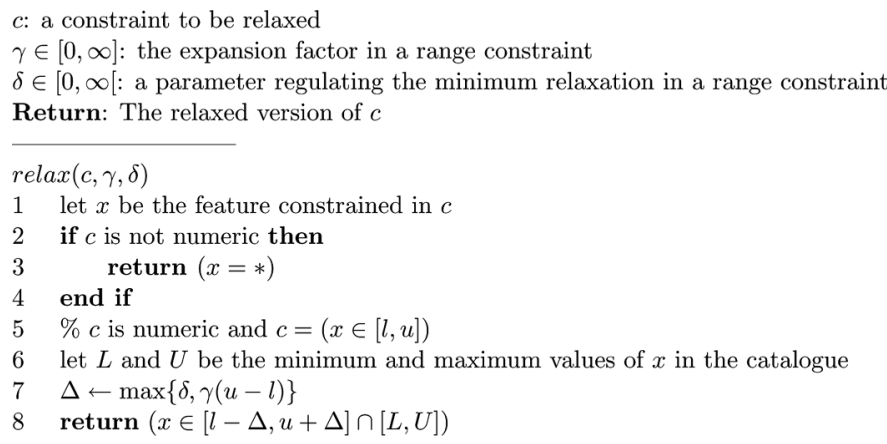

FIGURE 2 Single constraint relaxation.

Note that $\gamma$ and $\delta$ are feature dependent parameters and should be tuned with the user sensitivity to changes in that feature. For instance, we can assume that on a hotel price the minimum change in price that would make some difference to the user is 2 Euros, i.e., $\delta=2$. Hence, if the constraint $(x \in[50,60])$ is relaxed and $\gamma=0.1$, then instead of creating a new constraint as $(x \in[49,61])$, with a $10 \%$ relaxation on both endpoints, we generate the constraint $(x \in[48,62])$. This method works particularly in case the user inputs an equality constraint in the form of a range constraint; for instance, $x \in[48,48]$.

Having defined the notion of relaxed constraint, we can say that a query $q^{\prime}$ is called a relaxed subquery of $q$ if and only if for all $c^{\prime} \in q^{\prime}$ either $c^{\prime} \in q$ or exist $\gamma, \delta$ and $c \in q$ such that $c^{\prime}=\operatorname{relax}(c, \gamma, \delta)$.

Example 2. Let $q$ be the same query as in Example 1, then $q^{\prime}=\{[3 \leq$ category $\leq 4],[$ parking $=$ true $],[$ price $\leq 110],[$ restaurant $=$ true $]\}$ is a relaxed version of $q$, where the constraint on price is relaxed by 10 percent $(\gamma=0.1)$.

In the query relaxation procedure, which is presented next, we search for nonfailing relaxed subqueries that differ from the input query only in one constraint. This is because from one side we want to minimize the changes to the original user request, and from another side we must tame the combinatorial explosion of considering all the possible relaxations.

For this reason, when relaxing a single constraint does not repair a failing query, we try to remove two constraints, but only if these two constraints have at least an "approximate" dependency relationship. In this sense, the relaxation procedure makes use of the notion of feature abstraction hierarchy, i.e., a hierarchy among some features of an item space. For instance, let us consider again the Example 2. In this query, there are constraints on both category and price features. In our perspective, the category feature is more abstract than the price, since a constraint on the category is probably less 
informative when the user has already specified a constraint on the price, but the opposite is not true, since we may still have quite a large range of prices in the hotels of a given category. In fact, looking at the data, one can discover that the partition induced by the price, on the space of all the hotels, is approximately a subpartition of the category. In other words, the knowledge of the price greatly reduces the uncertainty over the category, i.e., the conditional entropy of the category given the price is low (MacKay 2003). In database theory, one typically considers the functional dependency between attributes, for instance, between a key and another attribute (O’Neil and O'Neil 2001). Here we are referring to an imperfect or approximated functional dependency.

A hierarchy relation can be used in the relaxation process in a natural way; when two features linked by a hierarchical relationship are both present in the query, then the relaxation could start from the constraint on the feature with the lowest abstraction level. If relaxing that constraint does not produce any result, then it could be removed, and the constraint on the feature at the next higher abstraction level is tried. In this way an abstraction hierarchy provides a heuristics for the relaxation process, i.e., to try first to relax the features with lowest abstraction, because this will make the lightest changes to a user query, and if this is not enough, to proceed to a higher level. Going back to the example, if both category and price are constrained, then try first to relax the price constraint and then, if this is not enough, to relax the constraint on category.

An abstraction hierarchy can be defined by either mining the data and extracting functional dependencies or relying on background knowledge provided by domain experts. Functional dependencies should be rare, and more often probabilistic/approximate functional relationships between the features would be found. In our applications of IQM, we have followed this second approach, but the proposed IQM relaxation is independent from the method used to generate the abstraction hierarchies, and therefore other methods can be applied as well. Let us now precisely describe the relaxation algorithm and how feature abstraction hierarchies are used.

First of all, we denote with $F A H=\left\{F^{1}, \ldots, F^{k}\right\}$ a set of feature abstraction hierarchies, where each $F^{i}=\left(x_{1}^{i}, \ldots, x_{n_{i}}^{i}\right)$ is an abstraction hierarchy, i.e., an ordered list of features in $X$ (comparable features). We mean that $x_{j}^{i}$ is more abstract than $x_{l}^{i}$ if and only if $j<l$, and there are no common features in two different $F^{i}$ s, i.e., features in different $F^{i}$ s are not comparable.

Figure 3 depicts the algorithm used in the relax module to find nonfailing relaxed queries of a failing query. The constraints in the query $q=\left\{c_{1}, \ldots, c_{m}\right\}$ are first partitioned in the set $C L=\left\{c l_{1}, \ldots, c l_{m^{\prime}}\right\}$, where each $c l_{i}$ is an ordered list of constraints of $q$, such that the features in $c l_{i}$ belong only to one abstraction hierarchy $F^{j}$ (line 1 ). If the constrained 
$q=\left\{c_{1}, \ldots, c_{m}\right\}:$ a query to be relaxed.

$F A H=\left\{F^{1}, \ldots, F^{k}\right\}$ : a set of feature abstraction hierarchies

Return: A suggestion list $S L=\left\{s_{1}, \ldots, s_{k}\right\}, k \leq m, s_{i}=\left(q_{i}, n_{i}\right)$, $q_{i}$ successful sub-query of $q$ and $n_{i}=\operatorname{count}\left(q_{i}\right)$

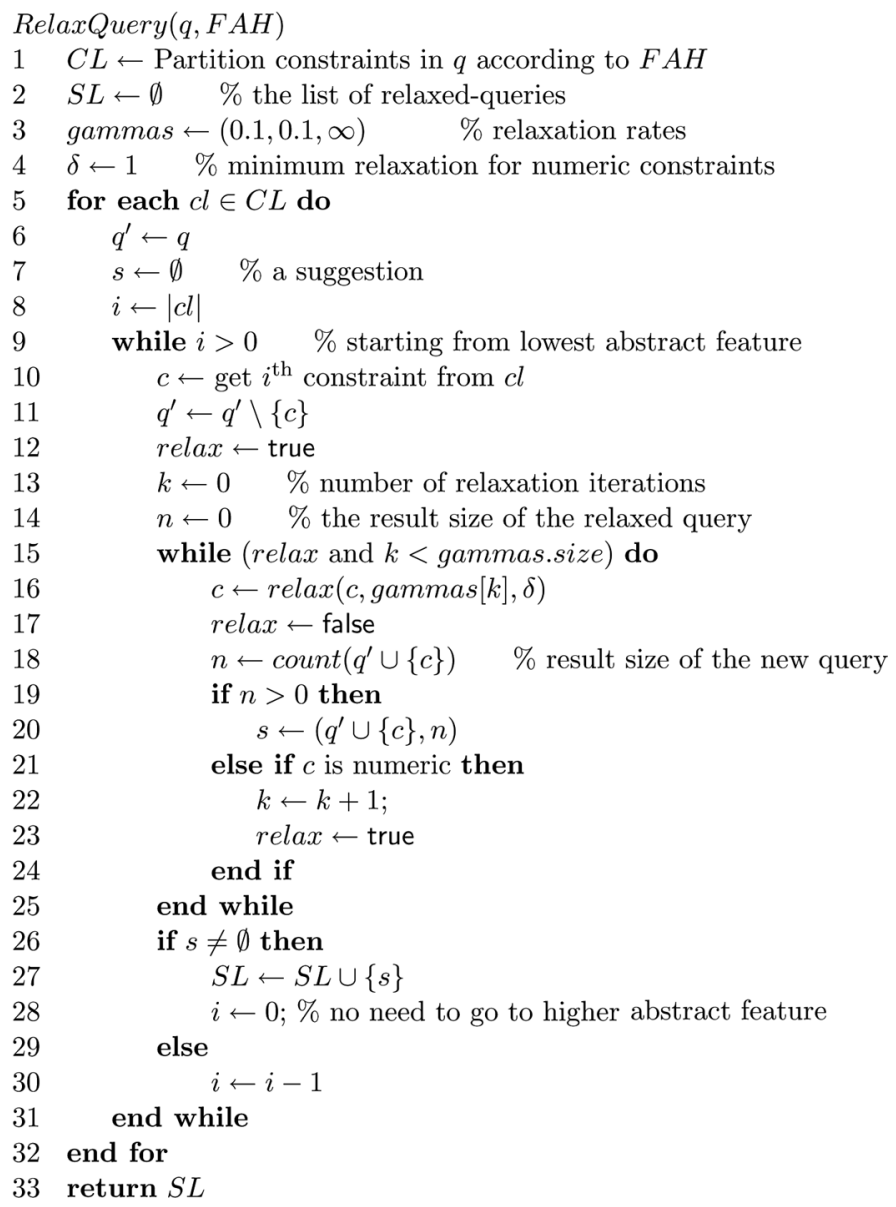

FIGURE 3 Query relaxation algorithm.

feature in $c$ does not belong to any abstraction hierarchy, then a list containing only the singleton $c$ is built.

Let us make an example of how a set of constraints in a query is partitioned according to a feature abstraction hierarchy. Let $q=\left\{c_{1}, c_{2}, c_{3}, c_{4}\right\}$ be the query as in Example 1, and let the feature abstraction hierarchy be $F A H=\{($ category, price $)\}$, then $C L=\left\{\left(c_{1}, c_{3}\right),\left(c_{2}\right),\left(c_{4}\right)\right\}$ is the resulting partition of $q$, according to this $F A H$. RelaxQuery outputs at most one relaxed query for each element in $C L$. The first relaxed query is obtained by relaxing the constraints in $\left(c_{1}, c_{3}\right)$, the second by relaxing $c_{2}$, and finally the third 
is obtained by relaxing $c_{4}$. To find the first relaxed subquery, the algorithm first checks if the relaxation of $c_{3}$ (price) is successful to produce some results, otherwise, it removes $c_{3}$ and relaxes $c_{1}$.

More precisely, first a list of relaxation rates is initialized at line 3 . The role of this array is to specify a sequence of $\gamma$ values by which a range constraint will be relaxed. The first and second entries are 0.1 , which means that a numeric constraint will be first relaxed with $\gamma=0.1$, then, if this is not producing a successful subquery, it will be further relaxed once more with $\gamma=0.1$ and then, finally, the constraint will be removed $(\gamma=\infty)$. Hence, a numerical constraint will be modified at most three times by the algorithm.

The loop at line 5 tries to relax the set of related constraints in each $c l \in C L$, while keeping the rest of the constraints in $q$ unchanged. The related constraints in $c l$ are relaxed starting from the constrained feature with the lowest abstraction by the while loop at line 9. $c$ is the constraint to be relaxed. The relax flag is used to control the loop of successive relaxations: only one relaxation for constraints on symbolic features, and a maximum number of times equal to gammas.size ( 3 in this case) for the numeric ones. The $n$ variable (line 14) contains the result size of the relaxed query.

The while loop at lines 15-25 tries to relax $c$, with different ranges for a numerical constraint, or it iterates only once for a non-numerical constraint. The range in a numerical constraint is modified, depending on the value of the current relaxation rate, i.e., gammas $[k]$. This loop terminates when the relaxation is successful, i.e., $n>0$ (line 19). In this case, the new relaxed query and the result size $n$ are inserted in the suggestion $s$ (line 20). If $n$ is still 0 , then a new relaxation is executed (in case the constraint $c$ is on a numeric feature).

At the end of this loop, if a suggestion has been found, then this is added to the suggestion list $S L$. Otherwise, a new constraint taken from the same abstraction hierarchy should be tried. At the end of this process, a suggestion list is returned. This contains the set (possibly empty) of relaxed queries and their relative result set sizes.

In fact, the relaxation algorithm we developed can also manage a situation that can occur in the execution of the RelaxQuery procedure, namely, that a numeric constraint is relaxed too much, producing a too large result set. In this case, before inserting the suggestion in the suggestion list (line 27), the system tries to reduce the range to a size that is in the middle between that causing the initial failure (empty result set) and the current too-large result set.

The running time of RelaxQuery is $O(|q|)$, where $|q|$ is the number of constraints in the query $q$, since each constraint is considered a constant number of times. 


\section{EMPIRICAL EVALUATION}

In this section, we present the evaluation of the IQM algorithms. We have analyzed the performance of the tighten and relax modules to understand how helpful they can be in assisting the decision maker (user), to solve the failure conditions we are considering. For the tighten module, we have compared the performance of the feature selection methods presented previously, in simulated user-system interactions. For the relaxation algorithm, we have evaluated the RelaxQuery algorithm on real failing queries, collected in a previous online evaluation of the NutKing recommender system with real users (Ricci et al. 2003).

\section{Feature Selection for Tightening}

In this section, we compare the performance of the four feature selection methods introduced. Each method first sorts, according to a score function, all features not yet included in the current query, and then it selects the top-scoring features. The score functions are: the Entropy, computed according to the feature values found in the current result set (Eq. (2)); the popularity (Eq. (3)); the utility (Eq. (7)); and random, as a baseline for the comparison, which assigns a random score to the feature.

These methods are compared by using a generic evaluation procedure that simulates user-system interactions. Each session is composed of some interactions. In each interaction, the system suggests a predefined number of features $\left(n_{f}\right)$, and the simulated user accepts, or not, at most one of them, to further constrain the initial query. The value provided by the (simulated) user for the selected feature is taken from a test item. Thus, a simulation basically models a user that is trying to select the test item.

The evaluation procedure is listed in Figure 4 in detail. The goal of this procedure is to run a set of simulated interactions and record the number of items in the result set after each simulated interaction $(\operatorname{count}(q)$ in the algorithm) and the popularity of the feature used in the interaction (not shown in the algorithm). This procedure takes as input a set of items (test set), the number of constraints $\left(n_{c}\right)$ in the initial test query that should be created on the base of the test item, the feature selection method $\lambda$ (i.e., one among entropy, popularity, random, and utility), the number of features $\left(n_{f}\right)$ to be suggested to the simulated user at each interaction, the acceptance probabilities of features $\mathbf{p}$, and a parameter iterate that, if true, forces the simulation to try $n_{f}$ additional features, if none of the previous $n_{f}$ were accepted by the simulated user.

At line 2, for each test item $(s)$, an initial query is generated, which randomly constrains one feature, among the four most popular features, to the value found in $s$. Then, if $n_{c}>1$, the rest of the required constraints are 


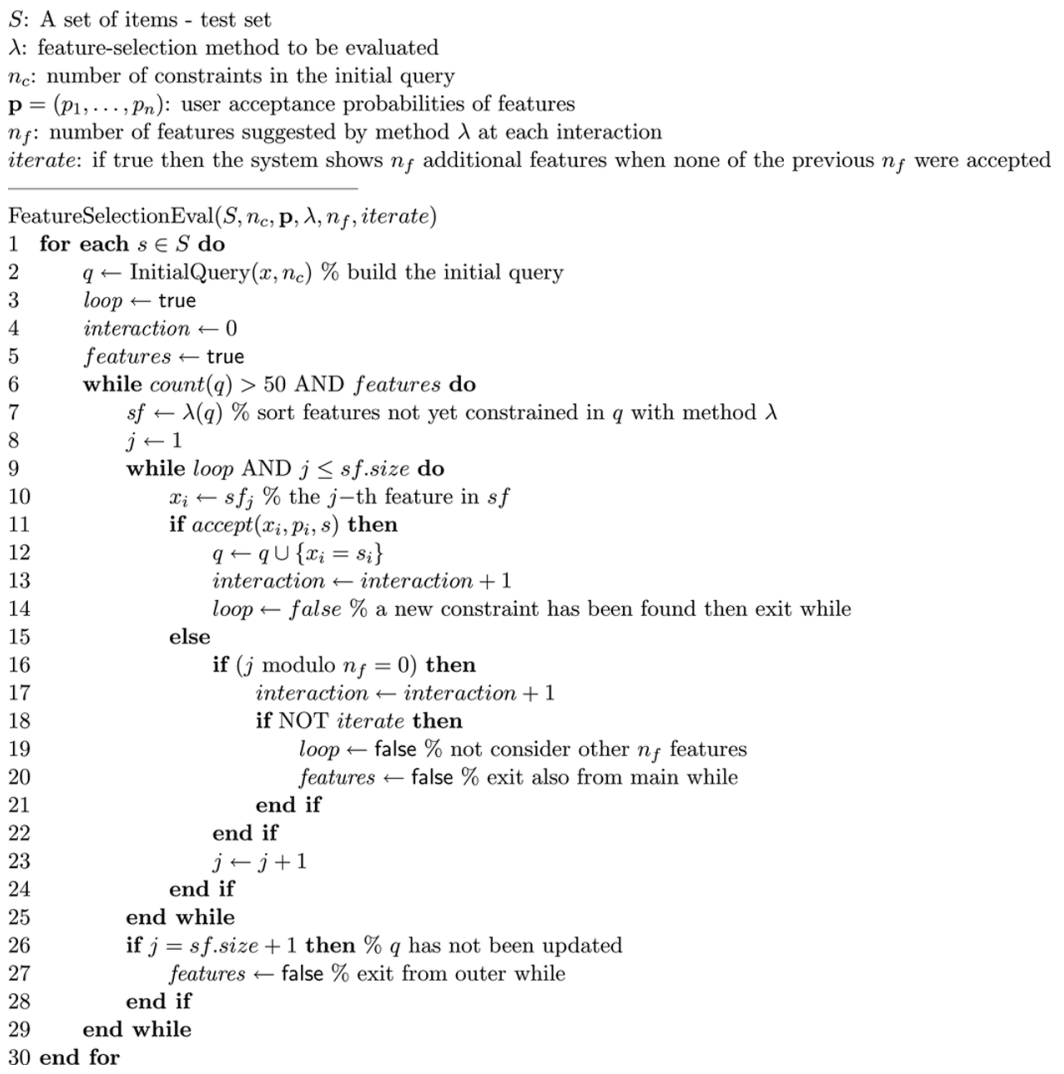

FIGURE 4 The feature selection evaluation procedure.

generated by choosing randomly $n_{c}-1$ features among those not constrained yet, and constraining them according to their values found in $s$. If the feature is numeric, then a range constraint is generated, i.e., a constraint of the form $x_{i} \in\left[l_{i}, u_{i}\right]$. In our simulation, the range is generated taking as lower (upper) bound $l_{i}\left(u_{i}\right)$ the value found in the test item $s_{i}$ and decreasing (increasing) it by $10 \%$, i.e., $l_{i}=0.9 s_{i}\left(u_{i}=1.1 s_{i}\right)$.

After this initial step, the loop starting at line 6 simulates the user-system interactions. If the result size of the query is above the given threshold (set to 50 in this algorithm), then the feature-selection method $\lambda$ is called to sort all the remaining features, i.e., those not constrained yet in $q$. Then the while loop at lines 9-25 simulates a set of interactions with the user, where the system suggests $n_{f}$ features per interaction and the user is supposed to accept the first that meets his interests.

In fact, at line 10, the first feature in $s f$ is selected and, at line 11 , there is the acceptance test. A feature $x_{i}$ is considered accepted by the simulated user with probability $p_{i}$, and its value $s_{i}$ in the test item $s$ is neither false (for 
Boolean features) nor null (not known), hence a meaningful constraint can be generated. If a feature is accepted, then we consider this an interaction, increasing the interaction counter and exiting from the inner while loop.

Conversely, if a feature is not accepted, we test if this is the last in a group of $n_{f}$ features (line 16). If this is the case, we increase the interaction counter. Moreover, if the iterate parameter is false, then we stop the simulation for test item $s$. This simulates a user who has not replied to any of the first $n_{f}$ (three in our experiments) suggestions and has aborted the IQM process. This is the interaction supported by NutKing. Then, we increment the feature counter $j$ in order to check a new feature, at the next iteration of the while loop (line 9).

The results of this evaluation algorithm depend on the feature selection method and on the probabilities $p_{i}$. If all of them are 1 , then this means that the user is always using a feature suggestion. Conversely, if these $p_{i}$ are smaller than one, then there is a chance that the simulated user will not reply, and the process can terminate anticipatively. These two situations are studied in the following sections.

In the two evaluations described in the next sections, we have considered an accommodations catalogue containing 3400 accommodations, ${ }^{1}$ modeled with 15 features: 2 numeric, and 13 Boolean.

\section{Evaluation 1: User Always Accepts Feature Suggestions}

Feature-selection methods for recommender systems have usually been evaluated with respect to the interactions length observed in simulated interactions (Aha and Breslow 1997; Kohlmaier et al. 2001; Shimazu 2001). A typical simulation proceeds by first selecting a target product (test item in the FeatureSelectionEval() algorithm), and then searching for those products that are most similar to the target with respect to a subset of all the product features. If the target product belongs to the first $k$ items retrieved (stop condition), then the procedure terminates. Otherwise, a new feature is selected, the target product provides the value for a new constraint on that feature, and a new retrieval is performed. The stop condition may vary, for instance a variant version stops the simulation when at least one product, among the 10 products most similar to the target, is found in the first $k$ items retrieved. All of these procedures iteratively add one feature after another to narrow the result set until the retrieval set contains an item considered as a good solution to the recommendation problem. The number of iterations is computed and better feature selection methods are considered those that produce shorter interactions.

As we have already mentioned, different from the quoted approaches that use similarity-based retrieval, we use-logical based filtering in which every item in the result set satisfies the query constraints, and we stop the 
simulation when the result set size is less than 50. Using logical-based filtering assures that the result set always contains the test item, since this satisfies all the queries built using the test item itself.

In this first experiment, we ran the evaluation procedure (Figure 4) on 500 items (the set $S$ ) randomly selected from the accommodation catalogue, with $n_{f}=3$, i.e., three features are supposed to be suggested to the user at each interaction and with probability one $(\mathbf{p}=\{1, \ldots, 1\})$ the user will use the first suggestion, if a value for that feature is present in the test item. Otherwise, the simulated user will try the second, and then the third. Moreover, the iterate parameter is set to true, i.e., if none of these three features have been accepted, then three more features are tried, until all the features are considered. Hence, we are following here a more traditional approach which assumes that a posed question is always replied by the user (Doyle and Cunningham 2000; Shimazu 2001; Bergmann and Cunningham 2002), and the simulation iterates until some feature can be used to tighten the current query.

Figure 5 shows the average result size of queries by different methods at each interaction and the average popularity of the feature used at a particular interaction. These results show that the entropy method outperforms the others, with respect to the reduction of the result size. This is somewhat an expected result, in line with previous evaluation. The major problem of the classical entropy method is that it selects features that are not "popular" among the users.

Conversely, the popularity and utility methods have better performance in term of popularity, which is quite obvious, but they cannot reduce the result size as fast as the entropy-based method. The popularity method performs even worse than the random one, from the fourth interaction. This happens because the features selected in the fourth to sixth interactions are correlated with those selected in the earlier interactions, and the newly

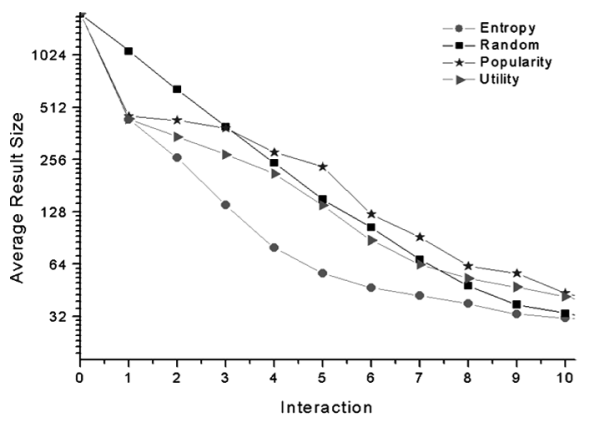

(a)

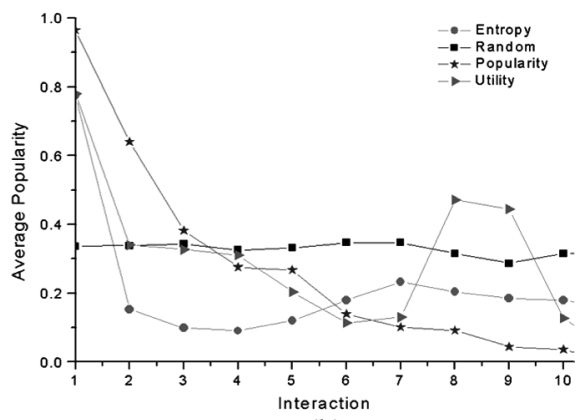

(b)

FIGURE 5 The average result size of queries at each interaction (a), and (b) the average popularity of used features at each interaction. 
built constraints are satisfied by most of the items in the catalogue. For instance, the parking feature is the fourth in terms of popularity, and $91 \%$ of accommodation items have a parking facility. We finally observe that the rise in average popularity (Figure 5(b)) at the eight and ninth interactions for the utility method is due to the fact that there are features with low utility, because the entropy factor $\left(U\left(S_{2 i-1}\right)=H_{R_{q}}^{i}\right.$ in Eq. (4)) is low, but the popularity of the feature is not.

The conclusion of this evaluation is that the classical entropy method is the best option when the user is supposed to always accept the suggested feature and constrain accordingly the result set. But what would happen if we explicitly model the fact that the user does not always reply to all question posed by the system? The following section presents the evaluation of the proposed feature selection methods, taking into account the likelihood of a user reply.

\section{Evaluation 2: User Does Not Always Accept Feature Suggestions}

In this section, we evaluate again the proposed feature selection methods, but with a different simulation of user-system interaction. Here we assume that with a nonzero probability, the user does not reply to the system feature suggestions, and if the initial set of suggested features (three in our example) are not accepted, then the simulated interaction is stopped.

As discussed previously, the probability $p_{i}$ that a user accepts a suggested feature, say $x_{i}$, can be estimated as $p_{i}=\beta \hat{p}_{i}$, for all $i=1, \ldots, n$. If we want to realistically simulate user behavior in an offline evaluation of the proposed feature selection methods, then we must estimate the constant $\beta$. The $\beta$ value must produce $p_{i}$ values that are close to the real acceptance rates that can be observed with human subjects.

It is worth mentioning that $\hat{p}_{i}$, i.e., the popularity of feature $x_{i}$, is the a-priori probability that a user constrains that feature in the initial query, and it is likely to be an overestimate of the acceptance probability of using $x_{i}$ in a successive interaction. This is because the more features the user has already constrained, the more unlikely it is that he further constrains other features.

In an experiment we conducted with real users, the entropy method was used in the tighten module, and we observed that the suggestions were accepted by the user only $28.6 \%$ of the times. This rather low acceptance rate can have many explanations. For instance, the graphical user interface might be too complicated and the user could not understand the suggestions, or the suggestions did not fall into the user's interests and were ignored. Whatever is the correct interpretation, this is irrelevant to our evaluation, provided that the simulated acceptance rate is close to that observed in real interactions. 
In order to estimate $\beta$ and obtain acceptance rates $\left(p_{i}=\beta \hat{p}_{i}\right)$ close to the observed ones, we ran simulations with different values of $\beta$, and we found that, with $\beta=0.5$, the overall acceptance rate of the entropy feature selection method comes close to $28.6 \%$. Then, we have used this acceptance model, i.e., $p_{i}=0.5 \hat{p}_{i}$, for all the methods, implicitly assuming that a feature is accepted or not with a probability that depends only on the feature itself and not on the other features suggested by a particular method (in previous interactions or in the same interaction).

We ran the evaluation procedure again with 500 randomly selected items, $n_{c}=1$ (one constraint in the initial query), $n_{f}=3$, i.e., only three features were suggested at each interaction, and iterate set to false. That last condition means that if none of the three suggested features is accepted, then the interaction stops. Finally, as we have mentioned, the acceptance model now is not trivial as before $\left(p_{i}=1\right)$, but $\mathbf{p}=\left(0.5 \hat{p}_{1}, \ldots, 0.5 \hat{p}_{n}\right\}$.

Figure 6 shows the results of the simulations under the new settings. The random method now performs worse than all the other methods for all interactions, which is much more meaningful than before. The pure entropy method does not reduce the result size much after the second interaction. On the other hand, the utility method, and more clearly the popularity method, which performed poorly in the previous simulations, are now the best. This is because they select features that may not be so effective in reducing the result set, but have a higher probability of being used, hence the simulated interaction proceeds. We must observe that, in these graphs, we assume that if a simulated interaction is stopped, for instance, at an interaction step with $\mathrm{M}$ items in the result set, then in the next interaction step, the result set is still of size M.

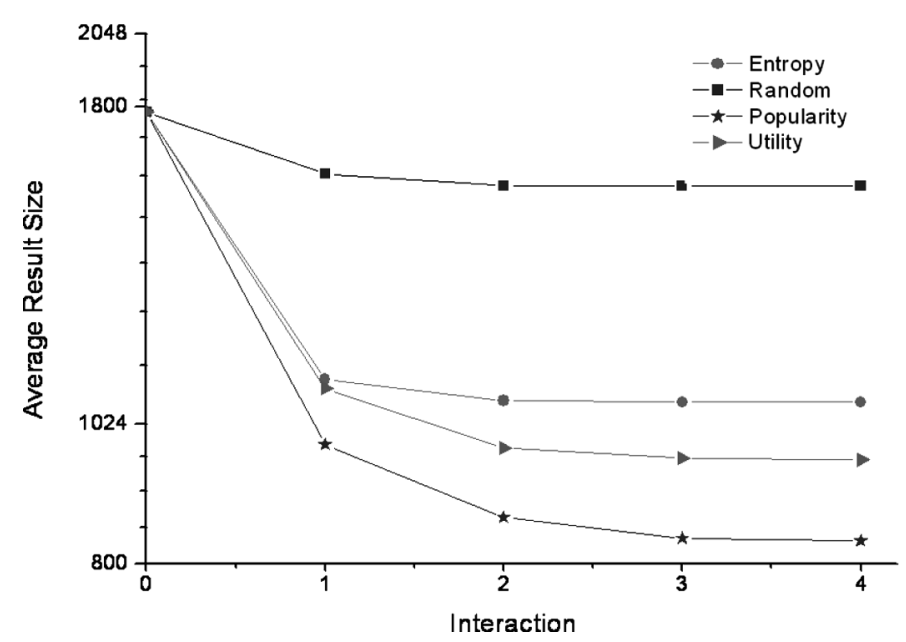

FIGURE 6 Average result size at each interaction with a probabilistic acceptance model. 
In a successive evaluation, we checked if the results shown before were due to a particular catalogue. Hence, we considered two additional catalogues used in another recommender system DieToRecs (Fesenmaier et al. 2003; Ricci et al. 2006b). DieToRecs was described previously, and, as we did for NutKing, we mined the real queries made by a population of users that tried the system prototype, and we computed the statistics required to measure the popularity and utility score mechanisms. The two additional catalogues are here called DTR-Destination and DTR-Lodging. The former is a catalogue of 600 locations in South Tirol (Austria) and Trentino (Italy). The latter is a catalogue of 20870 accommodations in the same regions. These catalogues contain real items promoted by TisCover.com. Also, in these two trials, we saw that the entropy method performs worse than utility and popularity. Here, in these examples (Figure 7), popularity behaves worse than utility. Looking more deeply in the evaluation results, we found that the reason was that these methods had almost the same acceptance rate in the simulations. But, since the utility of a feature is proportional to its entropy, a high scored feature by the utility method has relatively large entropy (in the result set) and it is likely to reduce the result size of the query better than a feature highly scored by popularity. In other words, in these examples, the utility method can better balance usefulness of the feature, in terms of result set size reduction and probability of being used.

A final observation is related to the computational cost of these methods. Both the entropy and utility methods require to compute the entropy of the remaining features on the result set and this has a linear time cost in the number of items in the results set. Conversely, the popularity method is a constant time (inexpensive) method.

\section{Query Relaxation Evaluation}

In this section, we present the empirical evaluation of the RelaxQuery procedure. From a previous empirical evaluation of the NutKing recommender system with real users, we derived a set of real queries that failed to retrieve any item, because they were over constrained. We have therefore examined the performance of the relaxation algorithm on these queries, and we have measured in how many cases RelaxQuery was able to assist the decision maker, explaining the cause of the failure. We shall also show an estimation of how difficult it would be for a user to find a successful (i.e., non-empty result set) relaxed-query by relaxing a minimum number of constraints, without any help from the interactive query management (IQM).

The evaluation discussed here is based on four catalogues: accommodation, event, location, and sport activities. We collected all the users' queries submitted to different catalogues, during an empirical evaluation of the NutKing system with real users. Let's call $Q$ this query set, and let 
TABLE 1 Queries Submitted to the Catalogues

\begin{tabular}{lrrrc}
\hline Catalogue & $Q$ & $F Q$ & $S Q$ & $S Q$ FAH \\
\hline Accommodation & 186 & 112 & 73 & 94 \\
Location & 116 & 64 & 29 & 50 \\
Event & 92 & 57 & 39 & 52 \\
Sport & 102 & 49 & 31 & 46 \\
Total & 496 & 279 & 172 & 232 \\
\hline
\end{tabular}

$\mathrm{Q}$ is the total number of queries, FQ is the subset of failing queries, SQ (SQ FAH) is the subset of FQ that can be successfully relaxed without (with) FAH.

$F Q \subseteq Q$ be the subset of failing queries, i.e., those returning a void result set. Then we ran the RelaxQuery algorithm on each query $q \in F Q$, to compare the subset of failing queries, say $S Q$, that the RelaxQuery could find a successful relaxed-query, with and without using the notion of featureabstraction-hierarchy (FAH) (Table 1). The relaxation algorithm that does not exploit FAH is described in Ricci et al. (2002), and is the algorithm used in the NutKing evaluation mentioned. This is basically similar to RelaxQuery presented here but does not group the query constraints according the FAH subsets, and hence the generated relaxations modify only a single constraint in the original failing query.

In total, (see Table 1) using $F A H$, the proposed algorithm was able to find a successful relaxed-query $83 \%$ of the cases (232/279), whereas the same algorithm not using $F A H$ was successful $61 \%$ (172/279). It is worth noting that this is obtained by losing a property of the previous method, i.e., the capability to find all the successful relaxed-queries that relax only one constraint. In other words, there are cases in which, using FAH, two constraints in a hierarchy are relaxed even when only one is necessary. This happens when relaxing the constraint on the more abstract feature, and

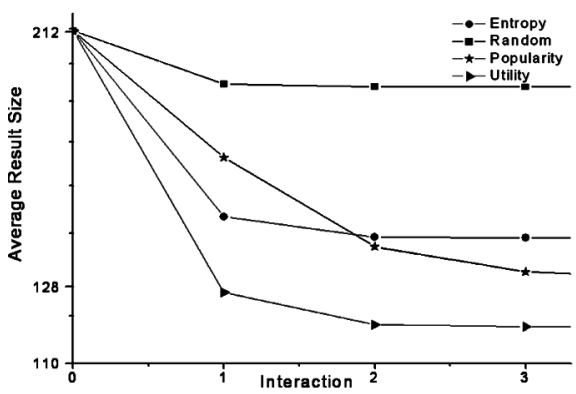

(a)

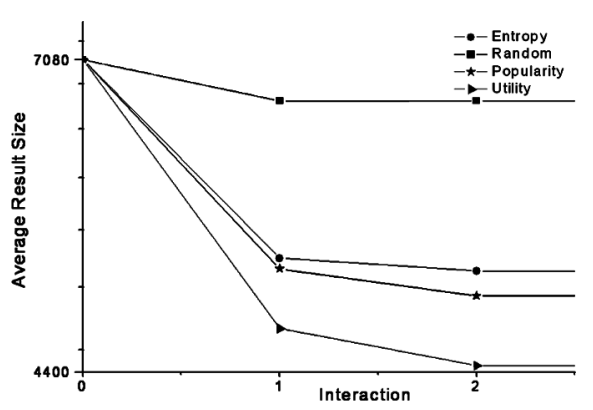

(b)

FIGURE 7 The average result size of queries at each interaction for two more data sets: (a) DTRDestination catalogue and (b) DTR-Lodging catalogue. 
still keeping the constraint on the less abstract feature would give some results. However, this situation is very unlikely since an abstraction relationship between $x$ and $y$ is an approximate functional dependency. In fact, let's assume that $y$ is functionally dependent from $x$, i.e., there is a function $f_{x y}$ from the domain of $x$ to the domain of $y$, such that $f_{x y}\left(v_{x}\right)=v_{y}$ for each tuple $v$ in the database, where $v[x]=v_{x}$ and $v[y]=v_{y}$ are the values of the attributes $x$ and $y$ in the tuple $v$. If we consider a query containing the constraints $y=v_{y}$ and $x=v_{x}$, with $v_{y}=f\left(v_{x}\right)$, then there cannot be a tuple $t$ such that the value of the more abstract feature does not satisfy the constraint, i.e., $t[y] \neq v_{y}$, and the value of the less abstract one satisfies the constraint, i.e., $t[x]=v_{x}$. Therefore, if the abstraction relationship is close to a functional dependency and the user is not generating queries in a random way, then our approach should keep the number of relaxations close to the minimum.

The Figures 8(a)-(d) compare the size of the sets $Q, F Q$, and $S Q$ for the location, accommodation, event, and sport catalogues, respectively, when $F A H$ is used. Figures $8(a)-(d)$ show that RelaxQuery can always find a

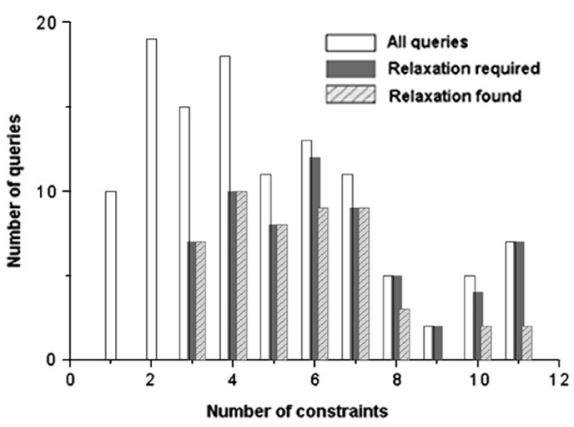

(a) Location

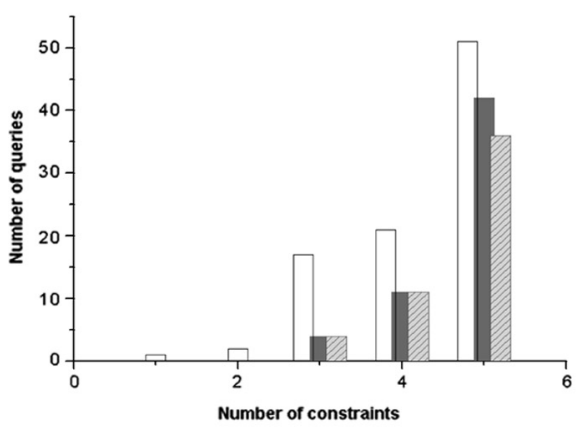

(c) Event

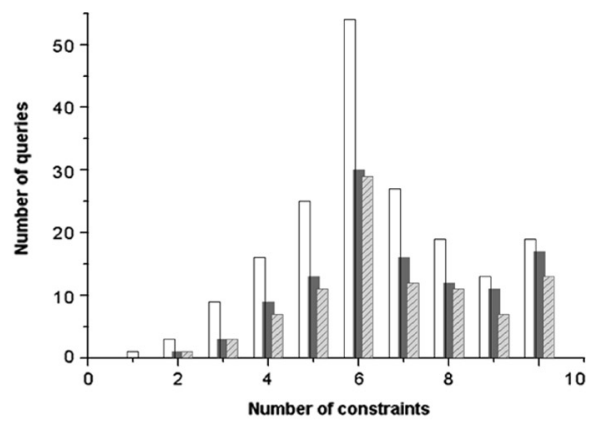

(b) Lodging

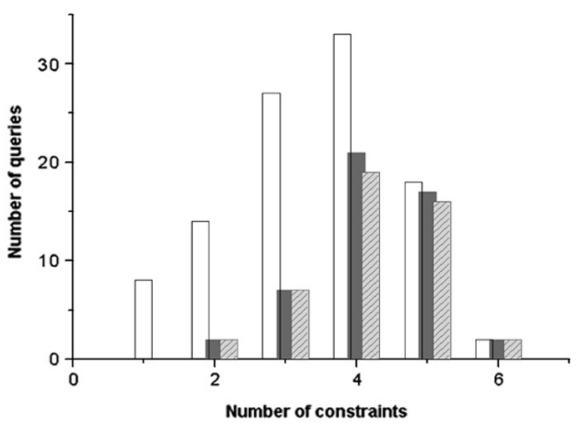

(d) Sport

FIGURE 8 RelaxQuery performance for different catalogues. 
successful subquery when the query (in our sample)contains up to three constraints. This number is even higher for the queries submitted to the event and lodging catalogues. In particular, considering the accommodation catalogue, we observe that more than half of the queries failed to return any item, and the RelaxQuery could find a subquery $83 \%$ of the time (Table 1).

Finally, we want to illustrate how difficult it would be for a user to refine autonomously her failing query. The discussion here is hypothetical, and it is aimed at computing, in the worst case scenario, the number of attempts required to repair a query by discarding a minimum number of constraints.

Let $q=\left\{c_{1}, \ldots, c_{m}\right\}$ be a query, and assume that the RelaxQuery method can find $k$ successful relaxed-queries. The maximum number of attempts the user has to make in order to find a successful change to her failing query, by relaxing only one constraint, is $m-k+1$. In fact, the user, after getting an empty result set, will remove one constraint, and she will submit the new query (second attempt). If this query fails again, then the user must put back the removed constraint and discard another one. This process will continue until the user receives, in the worst case, some results after $m-k+1$ attempts. Actually, this number will be even larger if a query constrains a numerical feature, and the user wants to try different ranges for that feature. This is obviously unfeasible in reality. A special case is when $k=1$; here $m$ attempts are required in the worst case. This is illustrated in Figure 9 with a dashed line. The straight line in the figure shows the actual average worst case $m-k+1$ computed on the sample of queries submitted to the accommodation catalogue during an empirical test. The dotted line

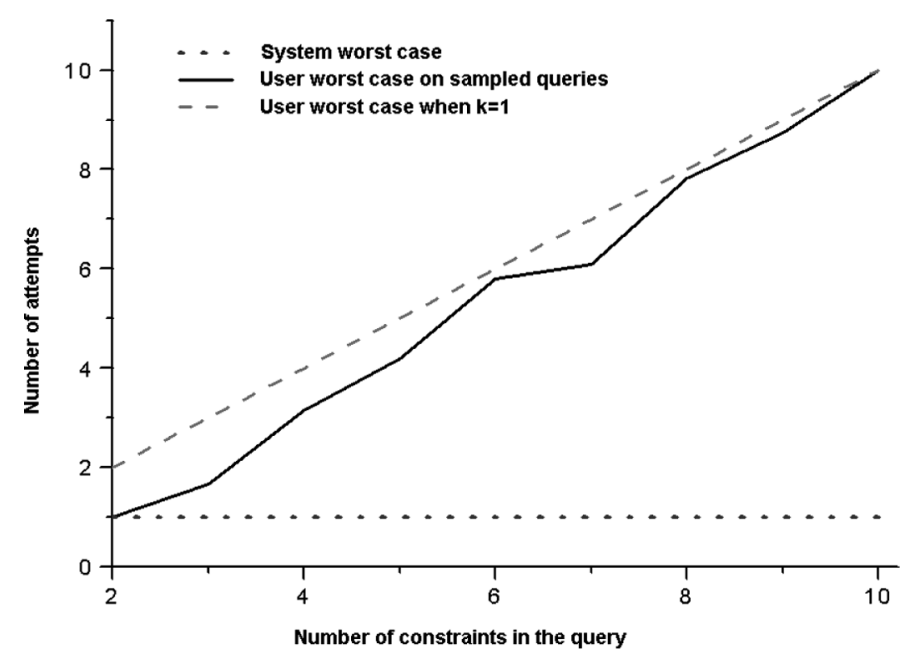

FIGURE 9 Attempts needed to find a relaxation ( $\mathrm{k}$ is the number of failing constraints). 
shows that a constant number of attempts is required if the user follows IQM suggestions.

\section{RECOMMENDER SYSTEM APPLICATIONS}

The interactive query management technology described in this article has been applied in two recommender systems: NutKing and DieToRecs. These systems provide recommendation for single products and for bundles of travel products (e.g., accommodation plus a destination and some event). These systems are driven by a recommendation methodology called Trip@dvice, which integrates case-based reasoning with interactive query management (Ricci et al. 2003; Ricci et al. 2006a). Case-based reasoning $(\mathrm{CBR})$ is a problem-solving methodology that tries to solve a problem at hand by using the solutions of past similar problems. (Aamodt and Plaza 1994; Watson 1997; Bridge et al. 2006). We have applied CBR for ranking (recommending) products that explicitly meet a user's preferences (query), by deriving implicit preferences contained in previously stored user-system interactions (cases). To achieve this goal, first IQM is used to support the retrieval of a manageable set of items that meet completely the user's needs. Then, CBR is exploited for ranking higher those retrieved products that are more similar to those selected in similar search contexts. We illustrate in the following sections two prototypes built using this methodology, focusing on the usage of IQM.

\section{NutKing}

NutKing recommends travel products and services (items) that the regional tourism organization of Trentino (Italy) promotes (Ricci and Del Missier 2004). When a query to a product catalogue (e.g., accommodations) returns too many items (i.e., more than 15), NutKing suggests three features, and it asks the decision maker (user) to provide her desired values for some of those features. For instance, Figure 10 shows, on the left, the form where a user inputs search conditions, here for a 3- or 4-star category hotel with the price ranging from 18 to 42 euros in the Trento area. The system finds items satisfying the query, and in this case, suggests to the user to specify one or more features. Here the suggestions are: 1) TV, 2) close to the city center, and 3) if the user has pets (right side of Figure 10). The user can specify any of those three features, or constrain other features, or browse the full list of 16 accommodations.

In the following snapshot (Figure 11), we see that the user has further specified: TV, close to the city center, and restaurant. Note that the restaurant feature was not suggested by the system, but the user has added it anyway. The system computes again the result size of the new query 


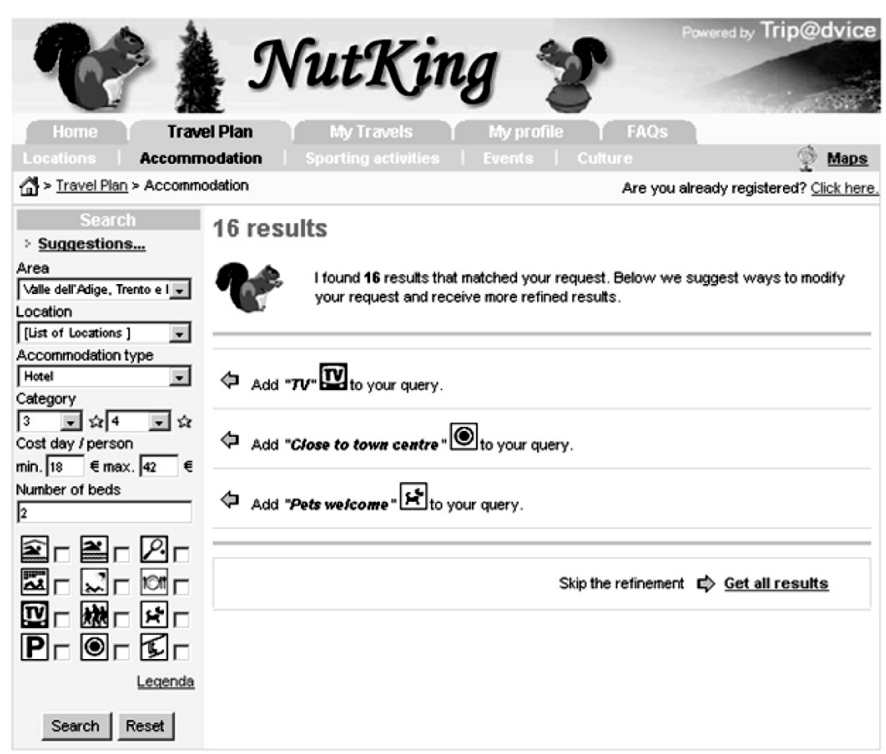

FIGURE 10 NutKing graphical user interface for query tightening.

and finds that there is no hotel satisfying the user's query. In this case, the system suggests to relax some constraints and explains that removing these preferences the user will get some results.

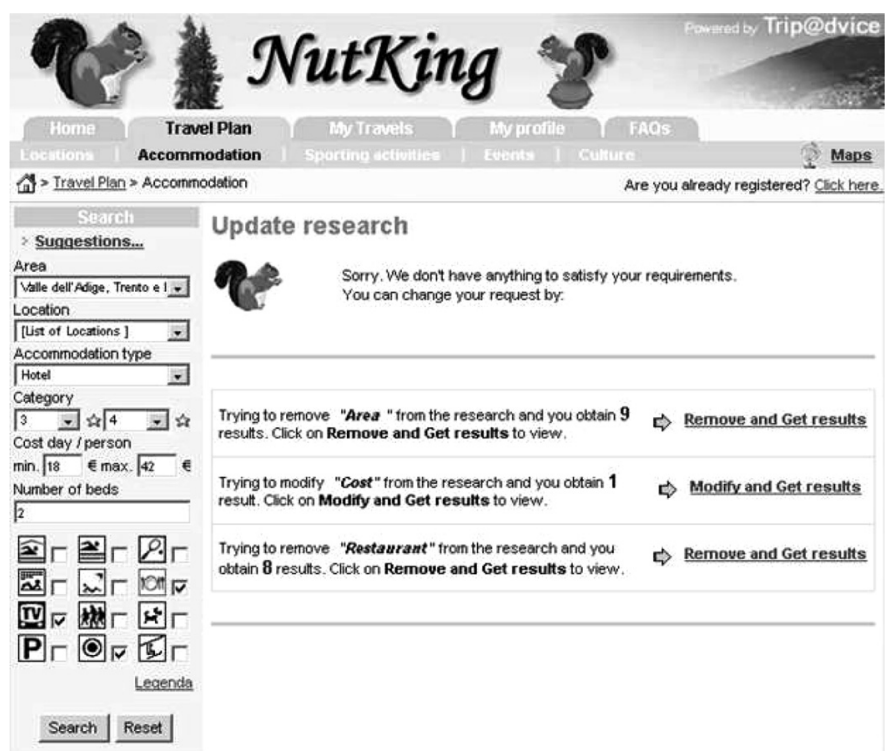

FIGURE 11 NutKing graphical user interface for query relaxation. 
We conducted an empirical evaluation of NutKing (see Ricci et al. [2003]), and we discovered some interesting results related to the interactive query management. We note that, in NutKing, the entropy method was used by the tighten module and the RelaxQuery method did not support the notion of feature abstraction hierarchy (FAH).

On average, 4.4 features were constrained in a user query and 13.4 queries were issued by a user in a recommendation session. For 6.3 queries (47\% of the total user queries), the system suggested some query relaxations, and in 2.8 cases ( $45 \%$ of the suggestions), the user accepted a suggested relaxation. We see this acceptance rate of the relaxation suggestions a good result, considering that the user behavior is often erratic and not always focused in solving the task.

On average, the system suggested in 2.1 of the user queries $(15.7 \%)$ to tighten the query, and only in 0.6 queries $(28.6 \%$ of the suggestions) the user accepted to tighten the query using one system suggestion. We hypothesized that it was because of the entropy-based feature selection method, and this motivated the new feature selection methods proposed in this article. For instance, in the example shown in Figure 10, the system suggests to specify if the user has pets, because (roughly) half of the accommodations in the result set allows guests to bring their pets and half does not, hence producing a large entropy score for that feature. But a few guests do have pets and are interested in replying to that question.

\section{DieToRecs}

DieToRecs (Intelligent Recommendation for Tourist Destination Decision Making) is a second recommender system that uses IQM (Fesenmaier et al. 2003; Ricci et al. 2006b). DieToRecs extends NutKing and supports multiple decision styles, one based on interactive query management and ranking based on case-based reasoning, and another called recommendation-by-proposing (Ricci et al. 2005), first introduced by Shimazu in his ExpertClerk system (Shimazu 2001). Here we focus on IQM usage in DieToRecs.

Figure 12 shows the IQM in action, guiding the user to recover from a failing query that requires tightening. In that figure, it is shown the system reply to a query for a 3- or 4-star category hotel and price in the range 18 to 42 Euros that is returning too many items. IQM suggests the additional features: boarding (i.e., breakfast or half board) and near city center. Note that, in DieToRecs, the user can select only one feature. If the query still returns a large result set, then three additional features are suggested. This process continues until the user either receives a small ranked set of items, or decides to look at all the items in the result set. If the user wants to add all the suggested features at once, then she has to follow the link to another page to edit her accommodation preferences (query). 


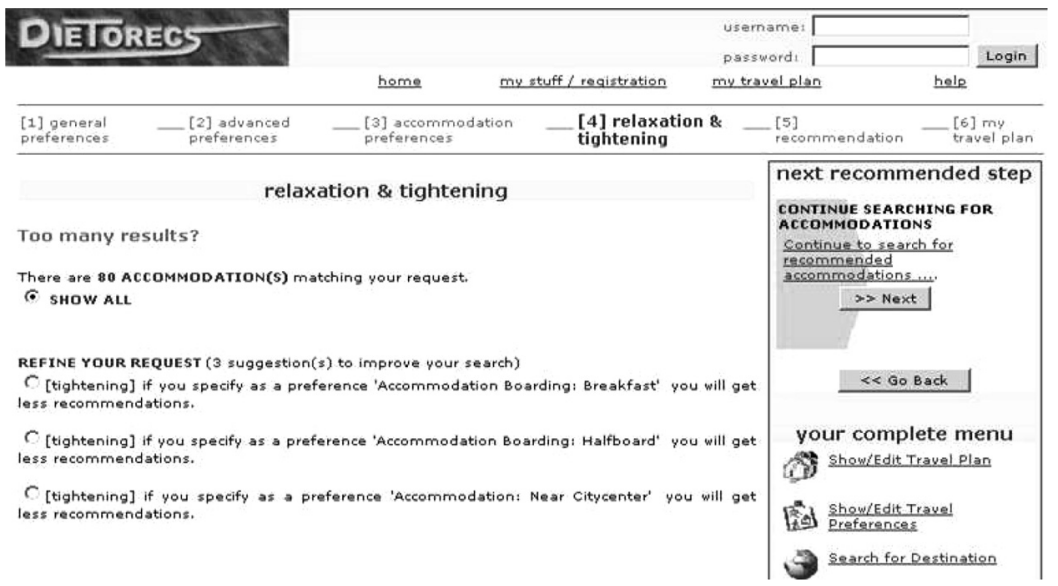

FIGURE 12 DieToRecs tightening user interface.

Figure 13 illustrates the situation where the user has added the features near city center, TV, and restaurant to the previous query. Now there is no hotel in the catalogue that satisfies the new query, and the system suggests to relax the TV feature, if the user wants to receive some results.

DieToRecs has been empirically evaluated with real users as well and compared with other systems. We refer the reader to Zins et al. (2004a) and (2004b), for more details on this evaluation.

\section{RELATED WORK}

Dealing with failing queries has been investigated largely in different studies either when a query returns a large result set, or when an empty

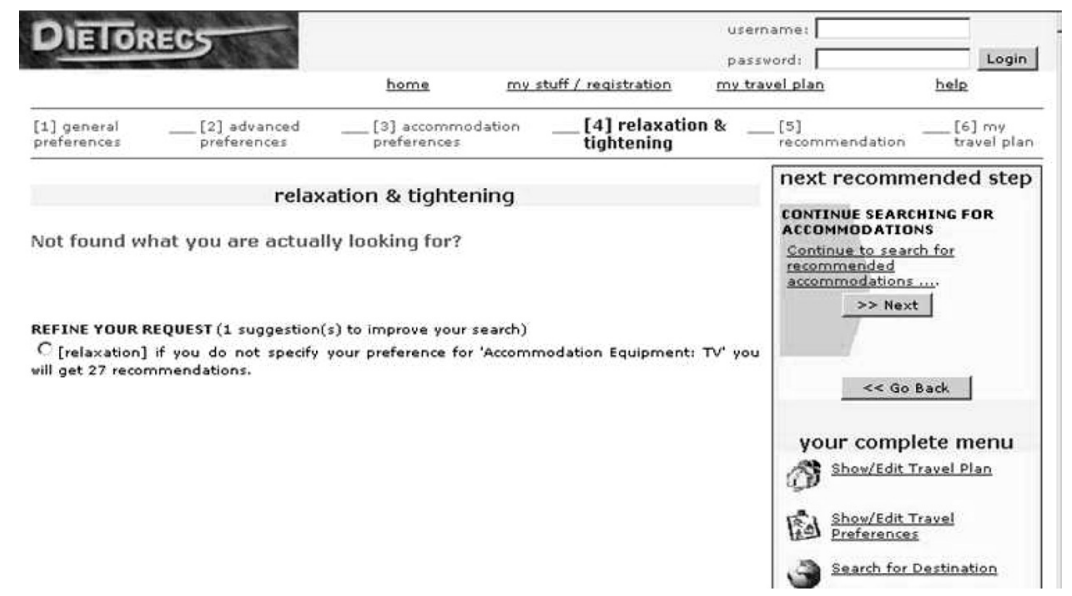

FIGURE 13 DieToRecs relaxation user interface. 
result set is found. Not much work has been dedicated to dealing simultaneously to both problems. Hence, we illustrate here some related research on feature selection techniques dealing with large result set queries, and research devoted to address the empty result set problem, which extend the discussion presented in the beginning of the article.

Much research has been dedicated to attribute-selection techniques in case-based reasoning. Most of the techniques were developed within CBR applications for diagnosis and classification, and more, in general, in conversational case-based reasoning systems (Göker and Thomson 2000; Gupta et al. 2002). In these applications, it is likely that the user would continue to answer the questions until she receives a solution to the problem. This seems not to apply in Web-based eCommerce applications, where the user is only one click away from a competing Web site and gets frustrated very easily if prompted with apparently unnecessary or not interesting questions. Thus, in eCommerce applications, the likelihood of a user reply has to be taken into account, and this has not been appropriately considered in the quoted works.

Doyle and Cunningham (2000) proposed an attribute selection technique for eCommerce applications based on (conceptual) clustering of the product catalogue. The idea is to select the feature at each step that best identifies a distinct cluster on the result set of the query. Shimazu (2001) has proposed an interaction model between a computerized sales clerk and a customer where the system can propose some products, in order to receive feedback from the user, or pose explicit questions selected, using an entropy-based scoring mechanism, until a manageable set of products is reached.

Our proposed feature selection method and its evaluation follows the work initiated by Kohlmaier et al. (2001). However, there are some differences. The most important is that they use similarity based retrieval, while, in our approach, a query is a logical filter containing a set of constraints that must be satisfied. Moreover, in our approach, the entropy of a feature is calculated on the result set of the query, while, in their approach, the computation is done on the whole catalogue. Both approaches try to simulate the question acceptance rate of the user, but the probability that a user will accept a tightening suggestion is estimated, in our case, by mining the log data of real user interactions. In their work, the knowledge of a domain expert is exploited. Other changes pertain to the precise evaluation strategy that simulate user-system interactions. We stop a simulated interaction if there is no reply to the posed questions (i.e., none of recommended features is accepted/used by the simulated user to further specify the query). In their simulation, the process continues until all questions have been answered. This approach, as we explained in this article, is clearly in favor of the entropy-based methods, but it is far from modeling the observed user behaviors. 
The research on queries with an empty result set goes back to the work of Kaplan (1982), where he describes a system that supports a cooperative query answering to queries expressed in natural language. He argues that it is more informative to get the user to understand the cause of failure, and to provide answers that partially satisfy the query, than just reporting a void answer like the empty result set.

Motro introduced the Flex system, a natural language front-end-toquery processing in relational databases (Motro 1990). Among many other properties, Flex knows how much to relax a numeric constraint. For instance, the constraint in $q_{0}=\{$ category $\leq 2\}$ must be relaxed by 1 , so when the query fails it is relaxed to $q_{1}=\{$ category $\leq 3\}$. In our methodology, numerical constraints are relaxed without using a-priori knowledge. Flex can compute all the minimal generalized failing queries. For example, if $q_{1}$ still returns no result, but $q_{2}=\{$ category $\leq 4\}$ returns some results, then $q_{1}$ is a minimal failing query of $q_{0}$. These queries are the most relaxed ones that still fail, hence provide an indication of the "cause" of the failure. This concept has been used also by McSherry (2003; 2005), which is discussed next. But unfortunately, finding all such minimal failing queries is an NPhard problem (Godfrey 1997). In contrast, we try to compute the maximal succeeding relaxed queries with the limitation of searching these queries only in a subset of all possible relaxations. The subset is given by the subqueries differing only on one constraint or in a group of constraints that are "approximately" functional dependent (abstraction hierarchy).

Value abstraction was introduced in the CoBase system (Chu et al. 1996), where an abstraction hierarchy among feature values was built, and the relaxation operators could move up and down in the hierarchy. In our approach, a hierarchy is a relationship between features (e.g., country $\succ$ county $\succ$ city), derived from domain knowledge or from the analysis of the data, and is exploited to find successful relaxed queries of a failing one. While building a values abstraction hierarchy is quite complex and costly in CoBase, in our approach, the feature hierarchy can be achieved with limited effort.

Gaasterland et al. describe a logic-based approach for developing a cooperative answering system that accepts natural language queries, and can explain the cause of any failing query (Gaasterland et al. 1992). Godfrey has extensively investigated the problem of identifying the cause of a failing Boolean query (Godfrey 1997). He has derived an algorithm (called ISHMAEL) to find successful maximal subquery (called XSS), i.e., those not contained in any other successful subquery. He shows that finding only one XSS can be done in linear time proportional to the number of constraints in $q$, but finding all XSS is intractable. The major difference between RelaxQuery and ISHMAEL relates to the search mode. While the latter does a depth-first-search in the query lattice to find an XSS, the former extends the lattice of subqueries, since the numerical constraints are not removed 
but relaxed to some extent, and the search is restricted to find queries that relax a minimum number of constraints. Hence, the latter is incomplete to avoid the combinatorial explosion of the search space.

McSherry (2003) has approached the relaxation problem differently by looking at the products that do not satisfy a given query. For each product in the catalogue, the set of attributes that do not satisfy the query (called compromise set) is computed. Products are grouped according to the compromises done, and a product/item (called case) representative of the compromise and having highest similarity to the query is put in a retrieval set. The problem with this approach is that the set of all possible compromises is exponential in the size of the query, and there is no indication about how to cope with such a problem.

Burke described a different approach to interactive query management called navigation by critiquing that implements a particular approach to automatic query relaxation (Burke 2000a; 2002b). The user starts the interaction with the system either mentioning a known product (restaurant), asking for a similar one, or selecting a set of high-level features (case features) and searching for a product that matches those features. With this input information, the system first selects from the database the set of all products that satisfy the largest number of logical constraints generated by considering the input features type and value. The system, if necessary, implicitly relaxes the least important constraints until some restaurants could be retrieved. Then the system sorts the retrieved cases using a similarity metric. This similarity metric assumes that the user goals, corresponding to the input features, could be sorted to reflect the importance of such goals from the user point of view. If the recommended product satisfies the user, then the interaction finishes. But, if the user is not satisfied, because of some features of the proposed product, then he can criticize them. If, for instance, the price is too high and the user is looking for something cheaper, then she can "tweak" the original request and provide a new input explicitly mentioning that the result must have a cheaper price. This starts a new recommendation cycle, and the criticized features are considered the most important user goal.

In this way, explicitly critiquing a feature means that the user's constraints on other product features will (implicitly) decrease their importance. In our approach, the user has complete control of the relaxation (and tightening) process with his preferred value(s), and at the same time, the system keeps the maximum number of the user's constraints in the relaxation process.

\section{CONCLUSIONS}

We have presented a front-end query processing methodology for recommender systems that copes with queries that return either a large result set or no results at all. 
Moving from an initial empirical evaluation (Ricci et al. 2003) that showed that the entropy-based feature selection technique suggests features not necessarily interesting for a user, we have introduced two new feature selection methods. The first assigns to each feature a score proportional to the probability of being used by the user, and the second assigns a utility value to a feature combining the feature information content and the probability of being used by the user. We have therefore compared these two methods with a previous one, the entropy-based one, in two types of simulated evaluations. The first one follows a more traditional approach that assumes the user always replies to a posed question/feature when he knows the value of the feature. In this case, the entropy-based method outperforms both proposed ones, because it selects features that effectively discriminate the underlying data source.

In application domains such as diagnosis, the assumption that a user always replies to a posed question is reasonable. But that assumption is not acceptable in eCommerce applications and in recommender systems where the user may not be interested in the additional product features proposed by the system (Anand and Mobasher 2005; Adomavicius and Tuzhilin 2005; Bridge et al. 2006). Therefore, in a second evaluation, we assigned to each feature an acceptance probability proportional to the feature popularity, measured in real user interactions with the system. Moreover, we tuned the overall acceptance rate in the simulations to be close to that measured in the user study. This second evaluation showed that, in cases where the probability of accepting a question is considered, the two new methods perform better in reducing the result size, because they select questions/features that will more likely be accepted, and therefore they keep the interaction alive. The proposed methods are very simple to implement, but they require some background knowledge of the user behavior, such as features' popularity and features' probabilistic dependency.

We have also presented in this article an approximate linear time algorithm for query relaxation. If a solution is found, then this is close to be maximal, with respect to the number of constraints kept in the successful relaxed query. Thus, since a query represents user's preferences, the algorithm relaxes as few of the user's preferences as possible. This algorithm is simple enough to be easily integrated into any eCommerce application. An empirical evaluation of the algorithm is here presented, and it is shown that it is powerful enough to recover from real failing situations most of the times $(86 \%$ of the input failing queries).

The current algorithms supporting the tightening and relaxation modules still have a number of limitations. As we have mentioned, the relaxation algorithm cannot find all the possible relaxations, and those found may not be the best. We plan to investigate other techniques that could find more relaxed successful subqueries not too far from the optimal ones 
(minimum constraints relaxation). This can only be achieved by exploiting heuristics driving the search for subsets of constraints to remove/relax, towards those that are the more likely cause of the failure. It is also important to note that not all the relaxations are equally preferable, hence an extended algorithm should integrate information about the more relevant or important features for the user.

This observation makes the relaxation and the tightening problems more close together. In fact, in both cases, we are investigating new ways to incorporate a user model into the relaxation/tightening algorithms. The more user preferences could be taken into account, the more likely it is that the proposed suggestions will be used, even if these are not optimal with respect to the minimization of the number of constraints relaxed or tightened. This makes this area an interesting and promising field for application of user modeling (Billsus and Pazzani 1997; Fisher 2001; Webb et al. 2001) and decision analysis (Clement 1996; Russell and Norvig 2003).

\section{REFERENCES}

Aamodt, A. and E. Plaza. 1994. Case-based reasoning: Foundational issues, methodological variations, and system approaches. AI Communications 7(1):39-59.

Adomavicius, G. and A. Tuzhilin. 2005. Toward the next generation of recommender systems: A survey of the state-of-the-art and possible extensions. IEEE Transactions on Knowledge and Data Engineering 17(6):734-749.Aha, D. W. and L. Breslow. 1997. Refining conversational case libraries. In: CaseBased Reasoning Research and Development, Proceedings of the 2 nd International Conference on Case-Based Reasoning (ICCBR-97), pages 267-278. Springer.

Anand, S. S. and B. Mobasher. 2005. Intelligent techniques for web personalization. In: Intelligent Techniques for Web Personalization, eds. Baushad Mobasher and Sarabjot Singh Anand, 1-36. Berlin, Germany: Springer.

Balabanovic, M. and Y. Shoham. 1997. Content-based, collaborative recommendation. Communication of ACM 40(3):66-72.

Bergmann, R. and P. Cunningham. 2002. Acquiring customers' requirements in electronic commerce. Artif. Intell. Rev. 18(3-4):163-193.

Billsus, D. and M. Pazzani. 1997. Learning probabilistic user model. In Proceedings of the Sixth International Conference on User Modeling (UM '97), Chia Laguna, Sardinia.

Billsus, D. and M. Pazzani. 1999. A hybrid user model for news story classification. In Proceedings of the Seventh International Conference on User Modeling (UM '99), pages 99-108, Banff, Canada.

Breese, J. S., D. Heckerman, and C. M. Kadie. 1998. Empirical analysis of predictive algorithms for collaborative filtering. In: Proceedings of the Fourteenth Conference on Uncertainty in Artificial Intelligence, pages 43-52, Madison, Wisconsin, USA: Morgan Kaufmann Publisher.

Bridge, D., M. Goker, L. McGinty, and B. Smyth. 2006. Case-based recommender systems. The Knowledge Engineering Review 20(3):315-320.

Bruno, N., S. Chaudhuri, and L. Gravano. 2002. Top-k selection queries over relational databases: Mapping strategies and performance evaluation. ACM Trans. Database Syst. 27(2):153-187.

Burke, R. 2000a. Knowledge-based recommender systems. In: Encyclopedia of Library and Information Science, eds. J. E. Daily, A. Kent, and H. Lancour, volume 69. New York: Marcel Dekker.

Burke, R. 2002b. Hybrid recommender systems: Survey and experiments. User Modeling and User-Adapted Interaction 12(4):331-370.

Chu, W. W., H. Yang, K. Chiang, M. Minock, G. Chow, and C. Larson. 1996. Cobase: A scalable and extensible cooperative information system. Journal of Intelligence Information Systems 6(2/3):223-259. 
Clement, R. T. 1996. Making Hard Decisions: An Introduction to Decision Analysis, 2nd ed. New York: Duxbury Press.

de Mantaras, R. L., D. McSherry, D. Bridge, D. Leake, B. Smyth, S. Craw, B. Faltings, M. L. Maher, M. T. Cox, K. Forbus, M. Keane, A. Aamodt, and I. Watson. 2006. Retrieval, reuse, revision and retention in case-based reasoning. Knowledge Engineering Review 20(3):215-240.

Doyle, M. and P. Cunningham. 2000. A dynamic approach to reducing dialog in on-line decision guides. In: Advances in Case-Based Reasoning, 5th European Workshop (EWCBR 2000), 49-60. Springer.

Fesenmaier, D. R., F. Ricci, E. Schaumlechner, K. Wöber, and C. Zanella. 2003. DIETORECS: Travel advisory for multiple decision styles. In: Information and Communication Technologies in Tourism 2003, eds. A. J. Frew, M. Hitz, and P. O’Connors, 232-241. Springer.

Fisher, G. 2001. User modeling in human-computer interaction. User Modeling and User-Adapted Interaction 11:65-86.

Gaasterland, T., P. Godfrey, and J. Minker. 1992. Relaxation as a platform for cooperative answering. Journal of Intelligent Information Systems 1(3/4):293-321.

Godfrey, P. 1997. Minimization in cooperative response to failing database queries. International Journal of Cooperative Information Systems 6(2):95-159.

Göker, M. H. and C. A. Thomson. 2000. Personalized conversational case-based recommendation. In: Proceedings of Advances in Case-Based Reasoning: 5th European Workshop (EWCBR-2000), pages 99-111, Trento, Italy, September 6-9, 2000. Springer.

Gupta, K. M., D. W. Aha, and N. Sandhu. 2002. Exploiting tax-onomic and causal relations in conversational case retrieval. In: Advances in Case-Based Reasoning, Proceedings of the 6th European Conference on Case Based Reasoning (ECCBR 2002), pages 133-147, Aberdeen, Scotland: Springer Verlag.

Kaplan, S. J. 1982. Cooperative responses from a portable natural language query system. Artificial Intelligence 19(2):165-187.

Kohlmaier, A., S. Schmitt, and R. Bergmann. 2001. A similarity-based approach to attribute selection in user-adaptive sales dialogs. In: Proceedings of the 4th International Conference on Case-Based Reasoning, volume 2080 of LNAI, pages 306-320, Vancouver, Canada: Springer.

Linden, G., B. Smith, and J. York. 2003. Amazon.com recommendations: Item-to-item collaborative filtering. IEEE Internet Computing 7(1):76-80.

Lorenzi, F. and F. Ricci. 2005. Case-based recommender systems: A unifying view. In: Intelligent Techniques for Web Personalization, IJCAI 2003 Workshop (ITWP 2003), 89-113, Acapulco, Mexico, August 11, 2003. Springer.

MacKay, D. J. C. 2003. Information Theory, Inference and Learning Algorithms, 1st ed. Cambridge: Cambridge University Press.

McSherry, D. 2003. Similarity and compromise. In: Proceedings of the 5th International Conference on CaseBased Reasoning (ICCBR 2003), pages 291-305, Trondheim, Norway.

McSherry, D. 2005. Retrieval failure and recovery in recommender systems. Artificial Intelligence Review 24(3-4):319-338.

Motro, A. 1990. Flex: A tolerant and cooperative user interface to databases. IEEE Transactions on Knowledge and Data Engineering 2(2):231-246.

MovieLens. 2007. Movielens web site. http://www. movielens.umn.edu.

O’Neil, P. and E. O'Neil. 2001. Database, Principles, Programming, and Performance. San Francisco, CA: Morgan Kaufmann.

Pazzani, M. J. 1999. A framework for collaborative, content-based and demographic filtering. Artificial Intelligence Review 13:393-408.

Quinlan, J. 1986. Induction of decision trees. Machine Learning 1:81-106.

Resnick, P., N. Iacovou, M. Suchak, P. Bergstrom, and J. Riedl. 1994. Grouplens: An open architecture for collaborative filtering of netnews. In: Proceedings ACM Conference on Computer-Supported Cooperative Work, pages 175-186.

Resnick, P. and H. R. Varian. 1997. Recommender systems. Communications of the ACM 40(3):56-58.

Ricci, F., D. Cavada, N. Mirzadeh, and A. Venturini. 2006a. Case-based travel recommendations. In: Destination Recommendation Systems: Behavioural Foundations and Applications, eds. D. R. Fesenmaier, K. Woeber, and H. Werthner, 67-93. Wallingford: CABI Publishing.

Ricci, F., D. R. Fesenmaier, N. Mirzadeh, H. Rumetshofer, E. Schaumlechner, A. Venturini, K. Wöber, and A. Zins. 2006b. DieToRecs: A case base travel advisory systems. In: Destination Recommendation 
Systems: Behavioural Foundations and Applications, eds. D. R. Fesenmaier, H. Werthner, and K. W. Wober, 227-239. Wallingford: CABI Publishing.

Ricci, F., N. Mirzadeh, and A. Venturini. 2002. Intelligent query managment in a mediator architecture. In: 2002 First International IEEE Symposium “Intelligent Systems," 221-226, Varna, Bulgaria.

Ricci, F. and F. Del Missier. 2004. Supporting travel decision making through personalized recommendation. In: Designing Personalized User Experiences in eCommerce, eds. C.-M. Karat, J. O. Blom, and J. Karat, 231-251. Dordrecht: Kluwer Academic Publisher.

Ricci, F., A. Venturini, D. Cavada, N. Mirzadeh, D. Blaas, and M. Nones. 2003. Product recommendation with interactive query management and twofold similarity. In: Proceedings of the 5 th International Conference on Case-Based Reasoning (ICCBR), eds. A. Aamodt, D. Bridge, and K. Ashley, pages 479-493, Trondheim, Norway.

Ricci, F., K. Woeber, and A. Zins. 2005. Recommendations by collaborative browsing. In: Information and Communication Technologies in Tourism 2005, 172-182. Wien-New York: Springer Verlag.

Russell, S. and P. Norvig. 2003. Artificial Intelligence: A Modern Approach, 2nd ed. Upper Saddle River: Prentice Hall.

Sarwar, K. G., J. Konstan, and J. Riedl. 2001. Item-based collaborative filtering recommendation algorithms. In: Proceedings of WWW 10 Conference, pages 285-295, Hong Kong. ACM.

Schafer, J. B., J. A. Konstan, and J. Riedl. 2001. E-commerce recommendation applications. Data Mining and Knowledge Discovery 5(1/2):115-153.

Shardanand, U. and P. Maes. 1995. Social information filtering: Algorithms for automating "word of mouth." In: Proceedings of the Conference on Human Factors in Computing Systems (CHI'95), pages 210-217.

Shimazu, H. 2001. ExpertClerk: Navigating shoppers buying process with the combination of asking and proposing. In: Proceedings of the Seventeenth International Joint Conference on Artificial Intelligence (IJCAI 2001), ed. B. Nebel, pages 1443-1448, Seattle, Washington, USA: Morgan Kaufmann.

van Rijsbergen, C. J. 1979. Information Retrieval. London: Butterworths, Available on-line http:// ftp.dcs.gla.ac.uk/Keith/Preface.html.

Watson, I. 1997. Applying Case-Based Reasoning: Techniques for Enterprise Systems. San Francisco, CA: Morgan Kaufmann.

Webb, G. I., M. J. Pazzani, and D. Billus. 2001. Machine learning for user modeling. User Modeling and User-Adapted Interaction 11:19-29.

Witten, I. H. and E. Frank. 2000. Data Mining. San Francisco, CA: Morgan Kaufmann Publisher.

Zins, A., U. Bauernfeind, F. D. Missier, N. Mitsche, F. Ricci, Il. Rumetshofer, and E. Schaumlechner. 2004a. Prototype testing for a destination recommender system: Steps, procedures and implications. In: Proceedings of the 11th International Conference on Information and Communication Technologies in Travel E् Tourism (ENTER 2004), ed. A. Frew, pages 249-258, Cairo, Egypt: Springer Verlag.

Zins, A., U. Bauernfeind, F. D. Missier, A. Venturini, and H. Rumetshofer. 2004b. An experimental usability test for different destination recommender systems. In: Proceedings of the 11th International Conference on Information and Communication Technologies in Travel E Tourism (ENTER 2004), pages 228-237, Cairo, Egypt: Springer Verlag.

\section{NOTE}

1. These are real accommodations promoted by the local tourist organization in Trentino region. 